\title{
Quenched local central limit theorem for random walks in a time-dependent balanced random environment
}

\author{
Jean-Dominique Deuschel ${ }^{1}(1) \cdot$ Xiaoqin Guo $^{2,3}$
}

Received: 6 December 2019 / Revised: 25 April 2021 / Accepted: 7 November 2021 /

Published online: 4 December 2021

(C) The Author(s) 2021

\begin{abstract}
We prove a quenched local central limit theorem for continuous-time random walks in $\mathbb{Z}^{d}, d \geq 2$, in a uniformly-elliptic time-dependent balanced random environment which is ergodic under space-time shifts. We also obtain Gaussian upper and lower bounds for quenched and (positive and negative) moment estimates of the transition probabilities and asymptotics of the discrete Green's function.
\end{abstract}

Keywords Parabolic Harnack inequality · Random walks in random environments · Non-divergence form operators · Balanced environments · Local limit theorem

Mathematics Subject Classification 60K37

\section{Contents}

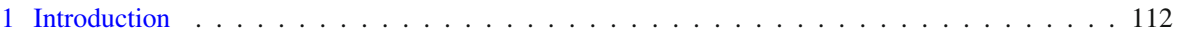

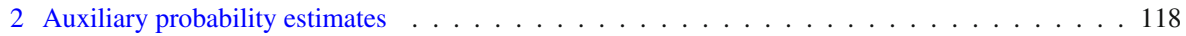

3 A local volume-doubling property and its consequences . . . . . . . . . . . . . 124

3.1 Volume-doubling properties . . . . . . . . . . . . . . . . . . . 125

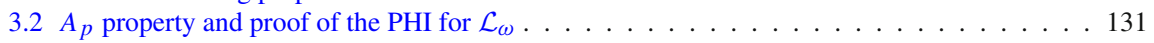

4 Estimates of caloric functions near the boundary . . . . . . . . . . . . . . . . . . . . 134

4.1 An elliptic-type Harnack inequality . . . . . . . . . . . . . . . . . . . . . 134

4.2 A boundary Harnack inequality . . . . . . . . . . . . . . . . . . 136

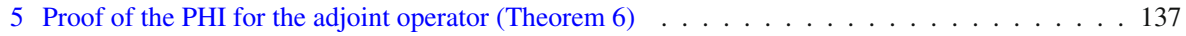

6 Proof of Theorems 4, 11, and Corollary $12 \ldots \ldots \ldots \ldots \ldots 14 \ldots \ldots \ldots$

$凶 \quad$ Jean-Dominique Deuschel deuschel@math.tu-berlin.de

Xiaoqin Guo guoxq@ucmail.uc.edu

1 Institüt für Mathematik, Technische Universität Berlin, Berlin, Germany

2 Department of Mathematics, University of Wisconsin-Madison, Madison, USA

3 Department of Mathematical Sciences, University of Cincinnati, Cincinnati, USA 
6.1 Proof of Theorem $11 \ldots \ldots$. . . . . . . . . . . . . . . . . . . . . . 141

6.2 Proof of Theorem 4 and Corollary $12 \ldots \ldots$. . . . . . . . . . . . . . . . 143

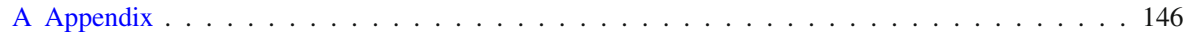

A.1 Properties (i)-(iii) in Remark 3 . . . . . . . . . . . . . . . . . . . . . . . . . 146

A.2 Proof of Corollary 7 . . . . . . . . . . . . . . . . . . . . . . . . . . . . . . . 147

A.3 Parabolic maximum principle . . . . . . . . . . . . . . . . . . . . . 148

A.4 Mean value inequality . . . . . . . . . . . . . . . . . . . . 150

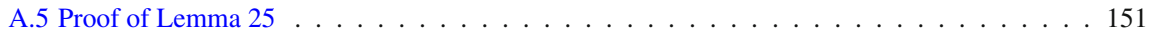

A.6 Proof of Corollary 12(ii)(iii) _ . . . . . . . . . . . . . . . . . . . . . 152

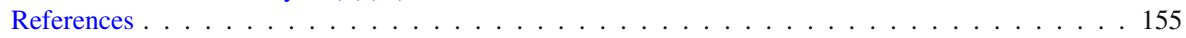

\section{Introduction}

In this article we consider a random walk in a balanced uniformly-elliptic timedependent random environment on $\mathbb{Z}^{d}, d \geq 2$.

For $x, y \in \mathbb{Z}^{d}$, we write $x \sim y$ if $|x-y|_{2}=1$. Denote by $\mathcal{P}$ the set (of nearestneighbor transition rates on $\mathbb{Z}^{d}$ )

$$
\mathcal{P}:=\left\{v: \mathbb{Z}^{d} \times \mathbb{Z}^{d} \rightarrow[0, \infty) \mid v(x, y)=0 \text { if } x \nsim y\right\} .
$$

Equip $\mathcal{P}$ with the the product topology and the corresponding Borel $\sigma$-field. We denote by $\Omega \subset \mathcal{P}^{\mathbb{R}}$ the set of all measurable functions $\omega: t \mapsto \omega_{t}$ from $\mathbb{R}$ to $\mathcal{P}$ and call every $\omega \in \Omega$ a time-dependent environment. For $\omega \in \Omega$, we define the parabolic difference operator

$$
\mathcal{L}_{\omega} u(x, t)=\sum_{y: y \sim x} \omega_{t}(x, y)(u(y, t)-u(x, t))+\partial_{t} u(x, t)
$$

for every bounded function $u: \mathbb{Z}^{d} \times \mathbb{R} \rightarrow \mathbb{R}$ which is differentiable in $t$. Let $\left(\hat{X}_{t}\right)_{t \geq 0}=$ $\left(X_{t}, T_{t}\right)_{t \geq 0}$ denote the continuous-time Markov chain on $\mathbb{Z}^{d} \times \mathbb{R}$ with generator $\mathcal{L}_{\omega}$. Note that almost surely, $T_{t}=T_{0}+t$. We say that $\left(X_{t}\right)_{t \geq 0}$ is a continuous-time random walk in the environment $\omega$ and denote by $P_{\omega}^{x, t}$ its law (called the quenched law) with initial state $(x, t) \in \mathbb{Z}^{d} \times \mathbb{R}$.

We equip $\Omega \subset \mathcal{P}^{\mathbb{R}}$ with the induced product topology and let $\mathbb{P}$ be a probability measure on the Borel $\sigma$-field $\mathcal{B}(\Omega)$ of $\Omega$. An environment $\omega \in \Omega$ is said to be balanced if

$$
\omega_{t}(x, x+e)=\omega_{t}(x, x-e) \quad \text { for all } e \in \mathbb{Z}^{d} \text { with }|e|=1 \text {. }
$$

and uniformly elliptic if there is a constant $\kappa \in(0,1)$ such that

$$
\kappa<\omega_{t}(x, y)<\frac{1}{\kappa} \quad \text { for all } t \in \mathbb{R}, x, y \in \mathbb{Z}^{d} \text { with } x \sim y .
$$

Let $\Omega_{\kappa} \subset \Omega$ denote the set of balanced and uniformly elliptic environments with ellipticity constant $\kappa \in(0,1)$. The measure $\mathbb{P}$ is said to be balanced and uniformly elliptic if $\mathbb{P}\left(\omega \in \Omega_{\kappa}\right)=1$ for some $\kappa \in(0,1)$. 
For each $(x, t) \in \mathbb{Z}^{d} \times \mathbb{R}$ we define the space-time shift $\theta_{x, t} \omega: \Omega \rightarrow \Omega$ by

$$
\left(\theta_{x, t} \omega\right)_{s}(y, z):=\omega_{s+t}(y+x, z+x) .
$$

We assume that the law $\mathbb{P}$ of the environment is translation-invariant and ergodic under the space-time shifts $\left\{\theta_{x, t}: x \in \mathbb{Z}^{d}, t \geq 0\right\}$. I.e., $P(A) \in\{0,1\}$ for any $A \in \mathcal{B}(\Omega)$ such that $\mathbb{P}\left(A \Delta \theta_{\hat{x}}^{-1} A\right)=0$ for all $\hat{x} \in \mathbb{Z}^{d} \times[0, \infty)$.

Given $\omega$, the environmental process

$$
\bar{\omega}_{t}:=\theta_{\hat{X}_{t}} \omega, \quad t \geq 0,
$$

with initial state $\bar{\omega}_{0}=\omega$ is a Markov process on $\Omega$. With abuse of notation, we use $P_{\omega}^{0,0}$ to denote the quenched law of $\left(\bar{\omega}_{t}\right)_{t \geq 0}$.

Assumptions: throughout this paper, we assume that $\mathbb{P}$ is balanced, ergodic, and uniformly elliptic with ellipticity constant $\kappa>0$.

We recall the quenched central limit theorem (QCLT) in [14].

Theorem 1 [14, Theorem 1.2] Under the above assumptions of $\mathbb{P}$,

(a) there exists a unique invariant measure $\mathbb{Q}$ for the process $\left(\bar{\omega}_{t}\right)_{t \geq 0}$ such that $\mathbb{Q} \ll \mathbb{P}$ and $\left(\bar{\omega}_{t}\right)_{t \geq 0}$ is an ergodic flow under $\mathbb{Q} \times P_{\omega}^{0,0}$. Let

$$
\rho(\omega):=\mathrm{d} \mathbb{Q} / \mathrm{d} \mathbb{P} .
$$

Then we have $\rho>0, \mathbb{P}$-almost surely, and

$$
E_{\mathbb{P}}\left[\rho^{(d+1) / d}\right]<\infty .
$$

(b) (QCLT) For $\mathbb{P}$-almost all $\omega, P_{\omega}^{0,0}$-almost surely, $\left(X_{n^{2} t} / n\right)_{t \geq 0}$ converges weakly, as $n \rightarrow \infty$, to a Brownian motion with deterministic non-degenerate covariance matrix $\Sigma=\operatorname{diag}\left\{2 E_{\mathbb{Q}}\left[\omega_{0}\left(0, e_{i}\right)\right], i=1, \ldots, d\right\}$.

In the special case where the environment is time-independent, i.e., $\mathbb{P}\left(\omega_{t}=\right.$ $\omega_{s}$ for all $\left.t, s \in \mathbb{R}\right)=1$, we say that the environment is static.

Remark 2 For balanced random walks in a static, uniformly-elliptic, ergodic random environment on $\mathbb{Z}^{d}$, the QCLT has been first shown by Lawler [24], which is a discrete version of the result of Papanicolaou and Varadhan [27]. It is then generalized to static random environments with weaker ellipticity assumptions in $[9,19]$.

Remark 3 Write $\|f\|_{L^{p}(\mathbb{P})}:=\left(E_{\mathbb{P}}\left[|f|^{p}\right]\right)^{1 / p}$ for $p \in \mathbb{R}$. At the end of the proof of [14, Theorem 1.2], it is shown that $E_{\mathbb{Q}}[g] \leq C\|g\|_{L^{d+1}(\mathbb{P})}$ for any bounded continuous function $g$, which implies (2).

For $(x, t) \in \mathbb{Z}^{d} \times \mathbb{R}$, set

$$
\rho_{\omega}(x, t):=\rho\left(\theta_{x, t} \omega\right) .
$$

Since $\Omega$ is equipped with a product $\sigma$-field, for any fixed $\omega \in \Omega$, the map $\mathbb{R} \rightarrow \Omega$ defined by $t \mapsto \theta_{0, t} \omega$ is measurable. Hence for almost-all $\omega$, the function $\rho_{\omega}(x, t)$ is measurable in $t$. Moreover, $\rho_{\omega}$ possesses the following properties. For $\mathbb{P}$-almost all $\omega$, 
(i) $\rho_{\omega}(x, t) \delta_{x} \mathrm{~d} t$ is an invariant measure for the process $\hat{X}_{t}$ under $P_{\omega}$;

(ii) $\rho_{\omega}(x, t)>0$ is the unique density (with respect to $\delta_{x} \mathrm{~d} t$ ) for an invariant measure of $\hat{X}$ that satisfies $E_{\mathbb{P}}\left[\rho_{\omega}(0,0)\right]=1$;

(iii) $\rho_{\omega}$ has a version which is absolutely continuous with respect to $t$ with

$$
\partial_{t} \rho_{\omega}(x, t)=\sum_{y} \rho_{\omega}(y, t) \omega_{t}(y, x)
$$

for almost every $t$, where $\omega_{t}(x, x):=-\sum_{y: y \sim x} \omega_{t}(x, y)$.

The proof of these properties, which is rather standard, is given in Sect. A.1 for the purpose of completeness.

As a main result of our paper, we will present the following local limit theorem (LLT), which is a finer characterization of the local behavior of the random walk than the QCLT. Let

$$
\hat{0}:=(0,0) \in \mathbb{Z}^{d} \times \mathbb{R}
$$

For $\hat{x}=(x, t), \hat{y}=(y, s) \in \mathbb{Z}^{d} \times \mathbb{R}, t \leq s$, define

$$
p^{\omega}(\hat{x}, \hat{y}):=P_{\omega}^{x, t}\left(X_{s-t}=y\right), \quad q^{\omega}(\hat{x}, \hat{y})=\frac{p^{\omega}(\hat{x}, \hat{y})}{\rho_{\omega}(\hat{y})} .
$$

Theorem 4 (LLT) For $\mathbb{P}$-almost all $\omega$ and any $T>0$,

$$
\lim _{n \rightarrow \infty} \sup _{x \in \mathbb{R}^{d}, t>T}\left|n^{d} q^{\omega}\left(\hat{0} ;\lfloor n x\rfloor, n^{2} t\right)-p_{t}^{\Sigma}(0, x)\right|=0
$$

Here $p_{t}^{\Sigma}(0, x)=\left[(2 \pi t)^{d} \operatorname{det} \Sigma\right]^{-1 / 2} \exp \left(-x^{T} \Sigma^{-1} x / 2 t\right)$ is the transition kernel of the Brownian motion with covariance matrix $\Sigma$ and starting point 0 , and $\lfloor x\rfloor:=$ $\left(\left\lfloor x_{1}\right\rfloor, \ldots,\left\lfloor x_{d}\right\rfloor\right) \in \mathbb{Z}^{d}$ for $x \in \mathbb{R}^{d}$.

The proof of the LLT follows from Theorem 1 and a localization of the heat kernel $q^{\omega}(\hat{0}, \cdot)$, an argument already implemented in [7] and [4] in the context of random conductance models. For this purpose, the regularity of $\hat{x} \mapsto q^{\omega}(\hat{0}, \hat{x})$ is essential. We use an analytical tool from classical PDE theory: the parabolic Harnack inequality (PHI) which yields not only Hölder continuity of $q^{\omega}(\hat{0}, \cdot)$ but also very sharp heat kernel estimates.

To state the PHI, we need some notations. For $\hat{x}=(x, t) \in \mathbb{Z}^{d} \times \mathbb{R}$, let

$$
\omega_{t}^{*}(x, y):=\frac{\rho_{\omega}(y, t) \omega_{t}(y, x)}{\rho_{\omega}(x, t)} \quad \text { for } x \sim y \in \mathbb{Z}^{d},
$$

and define the the adjoint operator $\mathcal{L}_{\omega}^{*}$ by

$$
\mathcal{L}_{\omega}^{*} v(\hat{x}):=\sum_{y: y \sim x} \omega_{t}^{*}(x, y)[v(y, t)-v(\hat{x})]-\partial_{t} v(\hat{x})
$$


We say that a function $u$ is $\omega$-caloric (resp. $\omega^{*}$-caloric) on $\mathcal{D} \subset \mathbb{Z}^{d} \times \mathbb{R}$ if $\mathcal{L}_{\omega} u=0$ (resp. $\mathcal{L}_{\omega}^{*} u=0$ ) on $\mathcal{D}$.

Throughout this paper, unless otherwise specified, $C, c$ denote generic positive constants that depend only on $(d, \kappa)$, and which may differ from line to line. If two functions $f$ and $g$ satisfy $c g \leq f \leq C g$, we write

$$
f \asymp g .
$$

First, let us state the PHI for the operator $\mathcal{L}_{\omega}$. For $r>0, x \in \mathbb{R}^{d}$, let

$$
B_{r}(x)=\left\{y \in \mathbb{Z}^{d}:|x-y|_{2}<r\right\}, \quad B_{r}=B_{r}(0) .
$$

Proposition 5 (PHI for $\mathcal{L}_{\omega}$ ) Assume $\omega \in \Omega_{\kappa}$ and $\theta>0$. Let $u$ be a non-negative $\omega$-caloric function on $B_{R} \times\left(0, \theta R^{2}\right)$. Then, for $0<\theta_{1}<\theta_{2}<\theta_{3}<\theta$, there exists a constant $C=C\left(\kappa, d, \theta_{1}, \theta_{2}, \theta_{3}, \theta\right)$ such that

$$
\sup _{B_{R / 2} \times\left(\theta_{2} R^{2}, \theta_{3} R^{2}\right)} u \leq C \inf _{B_{R / 2} \times\left[0, \theta_{1} R^{2}\right)} u .
$$

We remark that for dynamical environments in the discrete time setting, the PHI is obtained by Kuo and Trudinger for the so-called implicit form operators, see [23, (1.16)]. For discrete-time random walks in a static environment, the PHI is shown by Lawler [25] for uniformly elliptic operators, and by Berger and Criens [8] (see also [11]) for a genuinely $d$-dimensional i.i.d. environment which is not necessarily elliptic.

Observe that for fixed $\hat{x}=(x, t)$, the function $\hat{y} \mapsto q^{\omega}(\hat{y}, \hat{x})$ is $\omega$-caloric on $\mathbb{Z}^{d} \times(-\infty, t)$. Whereas, the heat kernel $\hat{x} \mapsto q^{\omega}(\hat{0}, \hat{x})$ is $\omega^{*}$-caloric on $\mathbb{Z}^{d} \times(0, \infty)$. Hence, to obtain the regularity of the heat kernel, we need to prove, instead of the PHI for $\mathcal{L}_{\omega}$, the following PHI for $\mathcal{L}_{\omega}^{*}$ which is our second main result.

Theorem 6 (PHI for $\mathcal{L}_{\omega}^{*}$ ) For $\mathbb{P}$-almost all $\omega$, any non-negative $\omega^{*}$-caloric function $v$ on $B_{2 R} \times\left(0,4 R^{2}\right]$ satisfies

$$
\sup _{B_{R} \times\left(R^{2}, 2 R^{2}\right)} v \leq C \inf _{B_{R} \times\left(3 R^{2}, 4 R^{2}\right]} v .
$$

As a standard consequence of the PHI for $\mathcal{L}_{\omega}^{*}$, we get the following Hölder estimate for $\omega^{*}$-caloric functions. (See a proof in Sect. A.2.)

Corollary 7 Let $\left(x_{0}, t_{0}\right) \in \mathbb{Z}^{d} \times \mathbb{R}$ and $R>0$. There exists $\gamma=\gamma(d, \kappa) \in(0,1]$ such that, $\mathbb{P}$-almost surely, any non-negative $\omega^{*}$-caloric function u on $B_{R}\left(x_{0}\right) \times\left(t_{0}-R^{2}, t_{0}\right]$ satisfies

$$
|u(\hat{x})-u(\hat{y})| \leq C\left(\frac{r}{R}\right)^{\gamma} \sup _{B_{R}\left(x_{0}\right) \times\left(t_{0}-R^{2}, t_{0}\right]} u
$$

for all $\hat{x}, \hat{y} \in B_{r}\left(x_{0}\right) \times\left(t_{0}-r^{2}, t_{0}\right]$ and $r \in(0, R)$. 
The main challenge in proving Theorem 6 is that $\omega^{*}$ is neither balanced nor uniformly elliptic, and so the PHI for $\mathcal{L}_{\omega}$ (Proposition 5) is not immediately applicable. This is the main difference with the random conductance model with symmetric jump rates where $\omega_{t}(x, y)=\omega_{t}(y, x)=\omega_{t}^{*}(x, y)$, in which case the PHI for $\mathcal{L}_{\omega}$ is the same as PHI for $\mathcal{L}_{\omega}^{*}$. See $[1,2,12,13,20]$.

In PDE, the Harnack inequality for the adjoint of non-divergence form elliptic differential operators was first proved by Bauman [6], and was generalized to the parabolic setting by Escauriaza [15]. Our proof of Theorem 6 follows the main idea of [15].

Let us explain the main idea for the proof of Theorem 6. An important observation is that $\omega^{*}$-caloric functions

can be expressed in terms of hitting probabilities of the time-reversed process. Thus to compare values of an

$\omega^{*}$-caloric function at different points, one only needs to estimate hitting probabilities of the original process that starts from the boundary. To this end, we will use a "boundary Harnack inequality" (Proposition 30) which compares

$\omega$-caloric functions near the boundary. We will also need the following parabolic volume-doubling property (VDP) for the invariant measure to control the change of probabilities due to time-reversal.

Theorem 8 (parabolic VDP) $\mathbb{P}$-almost surely, for every $r \geq 1 / 2$,

$$
\sup _{t:|t| \leq r^{2}} \rho_{\omega}\left(B_{2 r}, t\right) \leq C \rho_{\omega}\left(B_{r}, 0\right) \text {. }
$$

Remark 9 For time discrete random walks in a static environment, Theorem 6 was shown by Mustapha [26]. His argument follows basically [15], and uses the PHI [23, Theorem 4.4] of Kuo and Trudinger in the time discrete situation. Note that for static environments, the PHI for $\mathcal{L}_{\omega}^{*}$ follows from the PHI (for $\mathcal{L}_{\omega}$ ) and a representation formula (See Remark 33), and a VDP is not needed. However, in our dynamical setting, the parabolic VDP is crucially employed. To this end, we adapt ideas of Safonov-Yuan [28] and results in the references therein $[6,16,18]$ into our discrete space setting.

Remark 10 For adjoint solutions of non-divergence form elliptic PDE, a VDP was first established by Fabes and Stroock [17]. It was then generalized by Safonov and Yuan [28] to the parabolic case.

Recall the heat kernel $q^{\omega}$ in (4). For any $A \subset \mathbb{R}^{d}$ and $s \in \mathbb{R}$, let

$$
\rho_{\omega}(A, s)=\sum_{x \in A \cap \mathbb{Z}^{d}} \rho_{\omega}(x, s) .
$$

We write the $\ell^{2}$-norm of $x \in \mathbb{R}^{d}$ as $|x|=|x|_{2}$. For $r \geq 0, t>0$, define

$$
\mathfrak{h}(r, t)=\frac{r^{2}}{t \vee r}+r \log \left(\frac{r}{t} \vee 1\right) .
$$

Note that $\mathfrak{h}\left(c_{1} r, c_{2} t\right) \asymp \mathfrak{h}(r, t)$ for constants $c_{1}, c_{2}>0$. 
Our third main results are the following heat kernel estimates (HKE). Note that for a general balanced environment, the density $\rho_{\omega}$ does not have deterministic (positive) upper and lower bounds, thus one cannot expect deterministic Gaussian bounds for $p^{\omega}(\hat{0}, \hat{x})$. However, our HKE below shows that $p^{\omega}(\hat{0}, \hat{x})$ has both $L^{(d+1) / d}(\mathbb{P})$ and $L^{-p}(\mathbb{P})$ moment bounds.

Theorem 11 (HKE) For $\mathbb{P}$-almost every $\omega$ and all $\hat{x}=(x, t) \in \mathbb{Z}^{d} \times(0, \infty)$,

$$
\frac{c}{\rho_{\omega}\left(B_{\sqrt{t}}(y), s\right)} e^{-C \frac{|x|^{2}}{t}} \leq q^{\omega}(\hat{0}, \hat{x}) \leq \frac{C}{\rho_{\omega}\left(B_{\sqrt{t}}(y), s\right)} e^{-c \mathfrak{h}(|x|, t)}
$$

for all $s \in[0, t]$ and $y$ with $|y| \leq|x|+c \sqrt{t}$. Moreover, recalling the definition of $L^{p}(\mathbb{P})$ in Remark 3, there exists $p=p(d, \kappa)>0$ such that

$$
\begin{aligned}
\left\|P_{\omega}^{0,0}\left(X_{t}=x\right)\right\|_{L^{(d+1) / d}(\mathbb{P})} \leq \frac{C}{(t+1)^{d / 2}} e^{-c \mathfrak{h}(|x|, t)} \\
\text { and }\left\|P_{\omega}^{0,0}\left(X_{t}=x\right)\right\|_{L^{-p}(\mathbb{P})} \geq \frac{c}{(t+1)^{d / 2}} e^{-C \frac{|x|^{2}}{t}}
\end{aligned}
$$

for all $(x, t) \in \mathbb{Z}^{d} \times(0, \infty)$. As a consequence, setting $G^{\omega}(0, x)=\int_{0}^{\infty} P_{\omega}^{0,0}\left(X_{t}=\right.$ $x) \mathrm{d} t$, we have for $d \geq 3$ and $x \in \mathbb{Z}^{d}$,

$$
\left\|G^{\omega}(0, x)\right\|_{L^{(d+1) / d}(\mathbb{P})} \asymp\left\|G^{\omega}(0, x)\right\|_{L^{-p}(\mathbb{P})} \asymp(|x|+1)^{2-d} .
$$

Furthermore, we can characterize asymptotics of the Green's function of the RWRE. Recall the notations $\Sigma$ (in Theorem 1 (b)), $p_{t}^{\Sigma}$, and $\lfloor x\rfloor$ (in Theorem 4).

Corollary 12 The following statements are true for $\mathbb{P}$-almost every $\omega$.

(i) There exists $t_{0}(\omega)>0$ such that for any $\hat{x}=(x, t) \in \mathbb{Z}^{d} \times\left(t_{0}, \infty\right)$,

$$
\frac{c}{t^{d / 2}} e^{-\frac{C|x|^{2}}{t}} \leq q^{\omega}(\hat{0}, \hat{x}) \leq \frac{C}{t^{d / 2}} e^{-c \mathfrak{h}(|x|, t)}
$$

where $\mathfrak{h}$ is as in (5). As a consequence, the RWRE is recurrent when $d=2$ and transient when $d \geq 3$.

(ii) When $d=2$, for all $x \in \mathbb{R}^{d} \backslash\{0\}$,

$$
\lim _{n \rightarrow \infty} \frac{1}{\log n} \int_{0}^{\infty}\left[q^{\omega}(\hat{0} ; 0, t)-q^{\omega}(\hat{0} ;\lfloor n x\rfloor, t)\right] \mathrm{d} t=\frac{1}{\pi \sqrt{\operatorname{det} \Sigma}}
$$

(iii) When $d \geq 3$, for all $x \in \mathbb{R}^{d} \backslash\{0\}$,

$$
\lim _{n \rightarrow \infty} n^{d-2} \int_{0}^{\infty} q^{\omega}(\hat{0} ;\lfloor n x\rfloor, t) \mathrm{d} t=\int_{0}^{\infty} p_{t}^{\Sigma}(0, x) \mathrm{d} t .
$$


Similar results as Corollary 12(ii)(iii) are also obtained recently for the conductance model [5].

Our major technical novelties and main results can be summarized as follows. (a) Using probability estimates, we solve the difficult analytic problem of obtaining a parabolic VDP (for the density of the invariant measure) in a time-dependent balanced environment. (b) Using the parabolic VDP, we established the $A_{p}$ bounds, and as a consequence proved the PHI for $\omega$-caloric functions. The latter proof, which is of interest on its own, can be viewed as the parabolic version of Fabes and Stroock's [17] proof in the elliptic static setting. (c) Interpreting $\omega^{*}$-caloric functions in terms of a time reversed RWRE, and using the parabolic VDP and boundary PHI estimates, we prove the PHI for the adjoint operator. (d) As applications, we obtain LLT, quenched HKE, positive and negative $L^{p}$ bounds for the heat kernel, and Green's function asymptotics for the RWRE.

The organization of this paper is as follows. Section 2 contains probability estimates that are used in the later sections. In Sect. 3, we obtain the parabolic VDP and an $A_{p}$ inequality for $\rho_{\omega}$, and prove the PHI for $\mathcal{L}_{\omega}$. In Sect. 4 , we establish estimates of $\omega$-caloric functions near the boundary, showing both the interior elliptic-type and boundary PHI's. We prove the PHI for the adjoint operator (Theorem 6) in Sect. 5. Finally, with the adjoint PHI, we prove Theorems 4, 11, and Corollary 12 in Sect. 6. Some classical estimates and standard arguments can be found in the Appendix.

\section{Auxiliary probability estimates}

This section contains probability estimates that are crucial in the rest of the paper. For a finite subgraph $D \subset \mathbb{Z}^{d}$, let

$$
\partial D=\left\{y \in \mathbb{Z}^{d} \backslash D: y \sim x \text { for some } x \in D\right\}, \quad \bar{D}:=D \cup \partial D \text {. }
$$

For $\mathscr{D} \subset \mathbb{Z}^{d} \times \mathbb{R}$, define the parabolic boundary of $\mathscr{D}$ as

$$
\partial^{\mathrm{P}} \mathscr{D}:=\left\{(x, t) \notin \mathscr{D}:\left(B_{1+\epsilon}(x) \times(t-\epsilon, t]\right) \cap \mathscr{D} \neq \emptyset \text { for all } \epsilon>0\right\} .
$$

In the special case $\mathscr{D}=D \times[0, T)$ for some finite $D \subset \mathbb{Z}^{d}$, it is easily seen that $\partial^{\mathrm{P}} \mathscr{D}=(\partial D \times[0, T]) \cup(\bar{D} \times\{T\})$. See Fig. 1.

Recall the definition of the function $\mathfrak{h}(r, t)$ in $(5)$.

Lemma 13 Assume $\omega \in \Omega_{\kappa}$. Then for $t>0, r>0$,

$$
P_{\omega}^{0,0}\left(\sup _{0 \leq s \leq t}\left|X_{s}\right| \geq r\right) \leq C \exp (-c \mathfrak{h}(r, t)) .
$$

Proof Let $x(i), i=1, \ldots, d$, denotes the $i$-th coordinate of $x \in \mathbb{R}^{d}$. It suffices to show that for $i=1, \ldots, d$,

$$
P_{\omega}^{0,0}\left(\sup _{0 \leq s \leq t}\left|X_{S}(i)\right|>r\right) \leq C \exp (-c \mathfrak{h}(r, t)) .
$$


Fig. 1 The parabolic boundary of $D \times[0, T)$ time

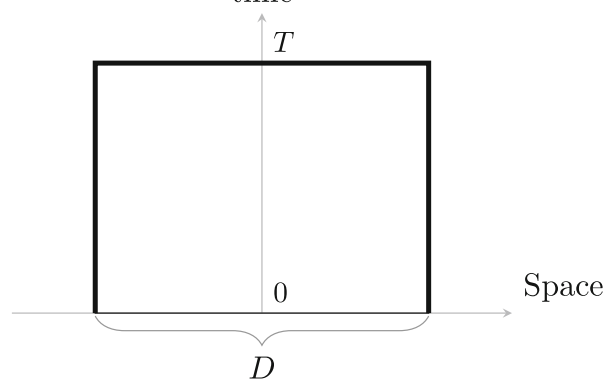

We will prove the statement for $i=1$. Let $\tilde{N}_{t}:=\#\left\{0 \leq s \leq t: X_{s}(1) \neq X_{s^{-}}(1)\right\}$ be the number of jumps in the $e_{1}$ direction before time $t$. Let $\left(S_{n}\right)$ be the discrete time simple random walk on $\mathbb{Z}$, then $X_{t}(1) \stackrel{d}{=} S_{\tilde{N}_{t}}$. Note that $\tilde{N}_{t}$ is stochastically dominated by a Poisson process $N_{t}$ with rate $c_{0}:=2 d / \kappa$, and so $P_{\omega}^{\hat{0}}\left(\sup _{0 \leq s \leq t}\left|X_{S}(1)\right|>r\right) \leq$ $P\left(\max _{0 \leq m \leq N_{t}}\left|S_{m}\right|>r\right)$. Hence,

$$
\begin{aligned}
P_{\omega}^{\hat{0}}\left(\sup _{0 \leq s \leq t}\left|X_{S}(1)\right|>r\right) & \leq P\left(N(t) \geq 2 c_{0}(t \vee r)\right)+P\left(\max _{0 \leq m \leq 2 c_{0}(t \vee r)}\left|S_{m}\right|>r\right) \\
& \leq e^{-c(t \vee r)}+C e^{-c r^{2} /(t \vee r)} \leq C e^{-c r^{2} /(t \vee r)}
\end{aligned}
$$

where we used Hoeffding's inequality in the second inequality. On the other hand, since the random walk is in a discrete set $\mathbb{Z}$, we have, for any $\theta>0$,

$$
\begin{aligned}
P_{\omega}^{\hat{0}}\left(\sup _{0 \leq s \leq t}\left|X_{S}(1)\right|>r\right) & \leq P(N(t)>r) \\
& \leq E[\exp (\theta N(t)-\theta r)]=\exp \left[c_{0} t\left(e^{\theta}-1\right)-\theta r\right] .
\end{aligned}
$$

When $r \geq 9 c_{0}^{2} t$, taking $\theta=\log \left(\frac{r}{c_{0} t}\right)$, we get an upper bound $\exp \left[-\frac{r}{2} \log \left(\frac{r}{t}\right)\right]$. Hence, letting $f(r, t)=\frac{r^{2}}{t \vee r} \mathbb{1}_{r<9 c_{0}^{2} t}+r \log \left(\frac{r}{t}\right) \mathbb{1}_{r \geq 9 c_{0}^{2} t}$, we obtain

$$
P_{\omega}^{\hat{0}}\left(\sup _{0 \leq s \leq t}\left|X_{S}(1)\right|>r\right) \leq C \exp (-c f(r, t)) .
$$

Since $f(r, t) \asymp \frac{r^{2}}{t \vee r}+r \log \left(\frac{r}{t}\right) \mathbb{1}_{r \geq 9 c_{0}^{2} t} \asymp \mathfrak{h}(r, t)$, our proof is complete.

Corollary 14 Assume $\omega \in \Omega_{\kappa}$ and $\theta_{2}>\theta_{1}>0$. There exist $C, c$ depending on $\left(d, \kappa, \theta_{1}, \theta_{2}\right)$ such that for $\theta \in\left(\theta_{1}, \theta_{2}\right),(x, t) \in \mathbb{Z}^{d} \times(0, \infty)$,

$$
P_{\omega}^{0,0}\left(X_{t} \in B_{\sqrt{\theta t}}(x)\right) \leq C \exp [-c \mathfrak{h}(|x|, t)]
$$

Proof Since $\mathfrak{h}(0, t)=0$, we only need to consider the case $x \neq 0$. 
If $\theta t \leq 1$, then $P_{\omega}^{\hat{0}}\left(X_{t} \in B_{\sqrt{\theta t}}(x)\right)=P_{\omega}^{\hat{0}}\left(X_{t}=x\right) \leq P_{\omega}^{\hat{0}}\left(\sup _{0 \leq s \leq t}\left|X_{s}\right| \geq|x|\right) \leq$ $C \exp [-c \mathfrak{h}(|x|, t)]$ by Lemma 13 .

If $\theta t>1$ and $1 \leq|x| \leq 2 \sqrt{\theta t}$, then $|x| \leq|x|^{2} \leq 4 \theta t$ and so $\mathfrak{h}(|x|, t) \asymp$ $\mathfrak{h}(|x|, 4 \theta t) \asymp \frac{|x|^{2}}{t}$. In particular, $\mathfrak{h}(|x|, t) \leq C \frac{|x|^{2}}{t} \leq c$. Hence, trivially, $P_{\omega}^{\hat{0}}\left(X_{t} \in\right.$ $\left.B_{\sqrt{\theta t}}(x)\right) \leq 1 \leq C \exp (-c h(|x|, t))$.

It reminds to consider $|x|>2 \sqrt{\theta t}>2$. In this case, by Lemma 13, $P_{\omega}^{\hat{0}}\left(X_{t} \in\right.$ $\left.B_{\sqrt{\theta t}}(x)\right) \leq P_{\omega}^{\hat{0}}\left(\sup _{0 \leq s \leq t}\left|X_{s}\right| \geq|x| / 2\right) \leq C \exp [-c \mathfrak{h}(|x|, t)]$.

For any $A \subset \mathbb{Z}^{d}, s \in \mathbb{R}$, define the stopping time

$$
\Delta(A, s)=\inf \left\{t \geq 0: \hat{X}_{t} \notin A \times(-\infty, s)\right\} .
$$

Lemma 15 Let $0<\theta_{1}<\theta_{2}, R>0$ and $\omega \in \Omega_{\kappa}$. Recall the stopping time $\Delta$ in (11). There exists a constant $\alpha=\alpha\left(\kappa, d, \theta_{1}, \theta_{2}\right) \geq 1$ such that for any $s \in\left(\theta_{1} R^{2}, \theta_{2} R^{2}\right)$ and $\sigma>0$

$$
\min _{x \in B_{R}} P_{\omega}^{x, 0}\left(X_{\Delta\left(B_{2 R}, s\right)} \in B_{\sigma R}\right) \geq\left(\frac{\sigma \wedge 1}{2}\right)^{\alpha} .
$$

Proof It suffices to consider the case $\sigma \in(0,1)$ and $R \geq K_{1}$, where $K_{1}=$ $K_{1}\left(\theta_{1}, \theta_{2}, \kappa, d\right)$ is a large constant to be determined. Indeed, if $R<K_{1}$, then by uniform ellipticity, for any $x \in B_{R}$,

$$
P_{\omega}^{x, 0}\left(X_{\Delta\left(B_{2 R}, s\right)} \in B_{\sigma R}\right) \geq P_{\omega}^{x, 0}\left(X_{s}=0, \Delta\left(B_{2 R}, s\right)=s\right)>C\left(\kappa, d, \theta_{1}, \theta_{2}\right) .
$$

Further, for $R \geq K_{1}$, if suffices to consider the case $\sigma R \geq \sqrt{K_{1}}$. Indeed, assume the lemma is proved for $R \geq K_{1}$ and $\sigma R \geq \sqrt{K_{1}}$. Then, when $\sigma R<\sqrt{K_{1}}$ and $x \in B_{R}$, by uniform ellipticity,

$$
\begin{aligned}
& P_{\omega}^{x, 0}\left(X_{\Delta\left(B_{2 R}, s\right)} \in B_{\sigma R}\right) \\
& \geq P_{\omega}^{x, 0}\left(X_{\Delta\left(B_{2 R}, s-K_{1}\right)} \in B_{\sqrt{K}_{1}}\right) \min _{y \in B_{\mathbb{K}_{1}}} P_{\omega}^{y, s-K_{1}}\left(X_{K_{1}}=0, \Delta\left(B_{2 R}, s\right)=K_{1}\right) \\
& \geq\left(\frac{\sqrt{K_{1}}}{2 R}\right)^{\alpha} C\left(K_{1}, \kappa, d\right) \geq C\left(\frac{\sigma}{2}\right)^{\alpha} .
\end{aligned}
$$

Hence in what follows we only consider the case $R \geq K_{1}$ and $\sigma R \geq \sqrt{K_{1}}$.

For $(x, t) \in \mathbb{R}^{d} \times \mathbb{R}$, set

$$
\psi_{0}(t)=1-\frac{1-(\sigma / 2)^{2}}{s} t, \quad \tilde{\psi}_{1}(x, t)=\psi_{0}-\frac{|x|^{2}}{4 R^{2}}, \quad \psi_{1}=\tilde{\psi}_{1} \vee 0,
$$

and, for some large constant $q \geq 2$ to be chosen,

$$
\psi(x, t):=\psi_{1}^{2} \psi_{0}^{-q}, \quad w(x, t)=(\sigma / 2)^{2 q-4} \psi(x, t) .
$$


Let $U:=\left\{\hat{x} \in B_{2 R} \times[0, s): \psi_{1}(\hat{x})>0\right\}$. We will show that for $\hat{x} \in U$,

$$
w(\hat{x}) \leq v(\hat{x}):=P_{\omega}^{\hat{x}}\left(X_{\tau} \in B_{\sigma R}\right) .
$$

Recall the parabolic boundary $\partial^{P}$ in (10). We first show that $w$ satisfies

$$
\left\{\begin{array}{l}
\left.w\right|_{{ }^{\mathrm{P}} U} \leq \mathbb{1}_{x \in B_{\sigma R}, s=t} \\
\min _{x \in B_{R}} w(x, 0) \geq \frac{1}{2}(\sigma / 2)^{2 q-4} \\
\mathcal{L}_{\omega} w \geq 0 \quad \text { in } U, \text { for } q \text { large. }
\end{array}\right.
$$

The first two properties in (12) are obvious. For the third property, note that

$$
\partial_{t} \psi=R^{-2} \psi_{0}^{-q}\left[\frac{1-(\sigma / 2)^{2}}{s / R^{2}}\left(q \frac{\psi_{1}}{\psi_{0}}-2\right) \psi_{1}\right] \quad \text { in } U
$$

For any unit vector $e \in \mathbb{Z}^{d}$, let

$$
\nabla_{e}^{2} u(x, t):=u(x+e, t)+u(x-e, t)-2 u(x, t) .
$$

When $\hat{x} \in U_{1}:=\{(z, s) \in U:(y, s) \in U$ for all $y \sim z\}$, then $\nabla_{e}^{2}\left[\psi_{1}^{2}(\hat{x})\right]=$ $\nabla_{e}^{2}\left[\tilde{\psi}_{1}^{2}(\hat{x})\right]$. When $\hat{x}=(x, t) \in U \backslash U_{1}$, then for some $|e|=1$, either $(x+e, t)$ or $(x-e, t)$ is not in $U$. Say, $(x+e, t) \notin U$, then $x \cdot e \geq 1$ and $\exists \delta \in(0,1)$ such that $\tilde{\psi}_{1}(x+\delta e, t)=0$. In both cases, there exists $\delta \in(0,1]$ such that

$$
\begin{aligned}
& \nabla_{e}^{2}\left[\psi_{1}(x, t)^{2}\right] \\
& =\tilde{\psi}_{1}^{2}(x+\delta e, t)+\tilde{\psi}_{1}^{2}(x-e, t)-2 \psi_{1}^{2}(\hat{x}) \\
& =-\frac{\left[1+\delta^{2}+2 x \cdot e(\delta-1)\right] \psi_{1}}{2 R^{2}}+\frac{1+\delta^{2}+4\left(\delta^{2}+1\right)(x \cdot e)^{2}+4\left(\delta^{3}-1\right) x \cdot e}{16 R^{4}} \\
& \geq-\frac{\psi_{1}}{R^{2}}+\frac{(x \cdot e)^{2}}{4 R^{4}}-\frac{\psi_{0}^{1 / 2}}{2 R^{3}},
\end{aligned}
$$

where in the last inequality we used the fact $1 \leq x \cdot e \leq|x| \leq 2 R \psi_{0}^{1 / 2}$. Thus, letting $\xi:=\psi_{1} / \psi_{0} \in[0,1]$, we have for $\hat{x}=(x, t) \in \tilde{U}$,

$$
\begin{aligned}
R^{2} \psi_{0}^{q-1} \mathcal{L}_{\omega} \psi(\hat{x}) & =R^{2}\left(\sum_{i=1}^{d} \omega_{t}\left(x, x+e_{i}\right) \nabla_{e_{i}}^{2}\left[\psi_{1}^{2}\right] / \psi_{0}+\psi_{0}^{q-1} \partial_{t} \psi\right) \\
& \geq \frac{c|x|^{2}}{R^{2} \psi_{0}}-C \xi-\frac{C}{R \psi_{0}^{1 / 2}}+\frac{1-(\sigma / 2)^{2}}{s / R^{2}}(q \xi-2) \xi \\
& \geq C q \xi^{2}-c_{1} \xi+c_{2}-c_{3} / K_{1}^{1 / 2}
\end{aligned}
$$


Fig. 2 The set $U$. The solid line is $\partial^{\mathrm{P}} U$

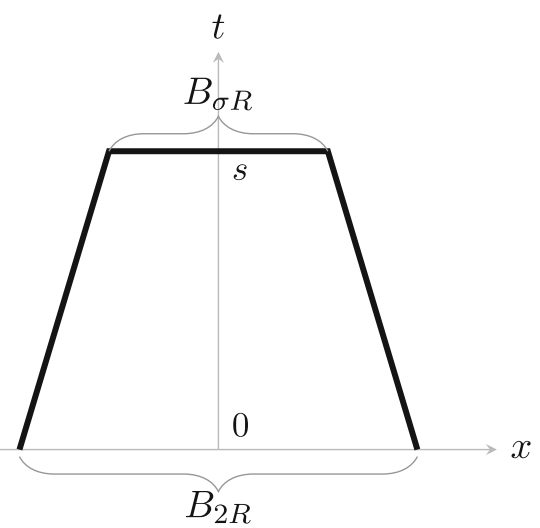

where in the last inequality we used $|x|^{2} /\left(4 R^{2} \psi_{0}\right)=1-\xi$ and $\psi_{0}^{1 / 2} \geq \sigma / 2 \geq$ $K_{1}^{1 / 2} /(2 R)$. Taking $q$ and $K_{1}$ large enough, we have $\mathcal{L}_{\omega} \psi \geq 0$ in $U$. The third property in (12) is proved.

Finally, we set $v(\hat{x})=P_{\omega}^{\hat{x}}\left(X_{\Delta\left(B_{2 R}, s\right)} \in B_{\sigma R}\right)$. By (12), $v\left(\hat{X}_{t}\right)-w\left(\hat{X}_{t}\right)$ is a supermartingale for $t \leq T_{U}:=\inf \left\{s \geq 0: \hat{X}_{s} \notin U\right\}$ and $\left.(v-w)\right|_{{ }^{\mathrm{P}} U} \geq 0$. Hence, the optional-stopping theorem yields

$$
v(\hat{x})-w(\hat{x}) \geq E_{a}^{\hat{x}}\left[v\left(\hat{X}_{T_{U}}\right)-w\left(\hat{X}_{T_{U}}\right)\right] \geq 0 \quad \text { for } \hat{x} \in U .
$$

In particular, $\min _{x \in B_{R}} v(x, 0) \geq \min _{x \in B_{R}} w(x, 0) \geq(\sigma / 2)^{2 q-4} / 2$.

Corollary 16 Assume that $\omega \in \Omega_{\kappa}, R / 2>r>1 / 2, \theta>0$. There exists $c=$ $c(d, \kappa, \theta) \in(0,1)$ such that for any $y \in \partial B_{R}$ with $B_{2 r}(y) \cap B_{R} \neq \emptyset$,

$$
\min _{x \in B_{r}(y) \cap B_{R}} P_{\omega}^{x, 0}\left(X \text {. exits } B_{2 r}(y) \cap B_{R} \text { from } \partial B_{R} \text { before time } \theta r^{2}\right)>c .
$$

Proof By uniform ellipticity, it suffices to consider $r \geq 10$.

Let $z=\frac{r y}{5|y|}+y \in \mathbb{R}^{d}$. Note that $B_{r}(y) \subset B_{5 r / 4}(z) \subset B_{3 r / 2}(z) \subset B_{2 r}(y)$ and $B_{r / 5}(z) \subset \mathbb{Z}^{d} \backslash B_{R}$. Recall $\Delta$ in (11). Then, by Lemma 15 ,

$$
\begin{aligned}
& \min _{x \in B_{r}(y) \cap B_{R}} P_{\omega}^{x, 0}\left(X_{\Delta\left(B_{2 r}(y) \cap B_{R}, \theta r^{2}\right)} \in \partial B_{R}\right) \\
& \geq \min _{x \in B_{5 r / 4(z)}} P_{\omega}^{x, 0}\left(X_{\Delta\left(B_{3 r / 2}(z), \theta r^{2}\right)} \in B_{r / 5}(z)\right) \geq c(\theta, d, \kappa) .
\end{aligned}
$$

The corollary is proved.

Lemma 17 Assume $\omega \in \Omega_{\kappa}, \beta \in(0,1)$. Let $\tau_{\beta, 1}=\tau_{\beta, 1}(R)=\inf \left\{t \geq 0: X_{t} \notin\right.$ $\left.B_{R} \backslash \bar{B}_{\beta R}\right\}$. Then if $y \in B_{R} \backslash \bar{B}_{\beta R} \neq \emptyset$ and $\theta>0$, we have

$$
P_{\omega}^{y, 0}\left(X_{\tau_{\beta, 1}} \in \partial B_{\beta R}, \tau_{\beta, 1} \leq \theta R^{2}\right) \geq C \frac{\operatorname{dist}\left(y, \partial B_{R}\right)}{R},
$$


where $C=C(\kappa, d, \beta, \theta)$.

Proof It suffices to prove the lemma for $R>\alpha^{2}$, where $\alpha=\alpha(\kappa, d, \beta, \theta)$ is a large constant to be determined. We only need to consider $y$ with $\operatorname{dist}\left(y, \partial\left(B_{R} \backslash \bar{B}_{\beta R}\right)\right) \geq 2$ in which case $R-|y| \asymp \operatorname{dist}\left(y, \partial B_{R}\right)$.

For $\hat{x}=(x, t)$, let $g(\hat{x})=\exp \left(-\frac{\alpha}{R^{2}}|x|^{2}-\frac{\alpha t}{\theta R^{2}}\right)$. Using the inequalities $e^{a}+e^{-a} \geq$ $2+a^{2}$ and $e^{a} \geq 1+a$, we get for $x \in B_{R} \backslash \bar{B}_{\beta R}, t \in \mathbb{R}$,

$$
\begin{aligned}
\mathcal{L}_{\omega} g(\hat{x}) & =g(\hat{x})\left(\sum_{i=1}^{d} \omega_{t}\left(x, x+e_{i}\right)\left[e^{-\frac{\alpha}{R^{2}}\left(1+2 x_{i}\right)}+e^{-\frac{\alpha}{R^{2}}\left(1-2 x_{i}\right)}-2\right]-\frac{\alpha}{\theta R^{2}}\right) \\
& \geq g(\hat{x})\left(\sum_{i=1}^{d} \omega_{t}\left(x, x+e_{i}\right)\left[e^{-\alpha / R^{2}}\left(2+4 \alpha^{2} x_{i}^{2} / R^{4}\right)-2\right]-\frac{\alpha}{\theta R^{2}}\right) \\
& \geq g(\hat{x})\left(-C \frac{\alpha}{R^{2}}+c \frac{\alpha^{2}|x|^{2}}{R^{4}}-\frac{\alpha}{\theta R^{2}}\right) \\
& \geq \frac{\alpha}{R^{2}} g(\hat{x})\left(c \alpha \beta^{2}-C\right)>0
\end{aligned}
$$

if $\alpha$ is chosen to be large enough. Hence $g\left(\hat{X}_{t}\right)$ is a submartingale for $t \leq \tau_{\beta, 1}$.

Recall the definition of the stopping time $\Delta$ in (11). Let

$$
v(\hat{x}):=\frac{g(\hat{x})-e^{-\alpha}}{e^{-\alpha \beta^{2}}-e^{-\alpha}} \quad \text { and } u(\hat{x}):=P_{\omega}^{\hat{x}}\left(X_{\Delta\left(B_{R} \backslash \bar{B}_{\beta R}, \theta R^{2}\right)} \in \partial B_{\beta R}\right)
$$

Set $\mathscr{D}=\left(B_{R} \backslash \bar{B}_{\beta R}\right) \times\left[0, \theta R^{2}\right)$. Since $\left.(u-v)\right|_{\partial^{\mathrm{P}} \mathscr{D}} \geq 0$ and $u\left(\hat{X}_{t}\right)$ is a martingale in $\mathscr{D}$, by the optional-stopping theorem we conclude that $u \geq v$ in $\mathscr{D}$. In particular, $u(x, 0) \geq v(x, 0) \geq C\left(R^{2}-|x|^{2}\right) / R^{2}$ for $x \in B_{R} \backslash \bar{B}_{\beta R}$.

Lemma 18 Let $\beta \in(0,1)$, and let $\tau_{\beta, 1}$ be as in Lemma 17. For $\theta>0$, there exists a constant $C=C(\beta, \kappa, d, \theta)$ such that, if $x \in B_{R} \backslash \bar{B}_{\beta R} \neq \emptyset$,

$$
P_{\omega}^{x, 0}\left(X_{\left(\theta R^{2}\right) \wedge \tau_{\beta, 1}} \notin \partial B_{R}\right) \leq C \operatorname{dist}\left(x, \partial B_{R}\right) / R
$$

Proof Set $\mathscr{D}:=\left(B_{R} \backslash \bar{B}_{\beta R}\right) \times\left[0, \theta R^{2}\right)$. It suffices to consider the case $R>k^{2}$, where $k=k(\beta, \kappa, d, \theta)>\log 2 / \log \left(2-\beta^{2}\right)$ is a large constant to be determined. Let $h(x, t)=2-|x|^{2} /(R+1)^{2}+t /\left(\theta R^{2}\right)$.

Recall the notation $\nabla^{2}$ in (13). For $\hat{x}=(x, t) \in \mathscr{D}$, note that $1 \leq h(\hat{x}) \leq 3$ and $\left|\nabla_{e_{i}}^{2}\left(h^{-k}\right)(\hat{x})-\partial_{i i}\left(h^{-k}\right)(\hat{x})\right| \leq C k^{3} R^{-3} h^{-k}(x, t)$. Hence for any $\hat{x}=(x, t) \in \mathscr{D}$, when $k$ is sufficiently large,

$$
\begin{aligned}
& \mathcal{L}_{\omega}\left(h^{-k}\right)(\hat{x}) \\
& \geq \sum_{i=1}^{d} \omega_{t}\left(x, x+e_{i}\right) \partial_{i i}\left(h^{-k}\right)-C k^{3} R^{-3} h^{-k}+\partial_{t}\left(h^{-k}\right)
\end{aligned}
$$




$$
\begin{aligned}
& \geq c \sum_{i=1}^{d}\left[k^{2} \frac{x_{i}^{2}}{(R+1)^{4}} h^{-k-2}+\frac{k}{(R+1)^{2}} h^{-k-1}\right]-C k^{3} R^{-3} h^{-k}-\frac{k}{\theta R^{2}} h^{-k-1} \\
& \geq c k h^{-k} R^{-2}\left[k-C-C k^{2} R^{-1}\right]>0,
\end{aligned}
$$

which implies that $h\left(\hat{X}_{t}\right)^{-k}$ is a submartingale inside the region $\mathscr{D}$.

Next, set (Recall the stopping time $\Delta$ in (11).)

$$
u(\hat{x})=P_{\omega}^{\hat{x}}\left(X_{\Delta\left(B_{R} \backslash \bar{B}_{\beta R}, \theta R^{2}\right)} \notin \partial B_{R}\right) .
$$

Then $u\left(\hat{X}_{t}\right)+\left(2-\beta^{2}\right)^{k} h\left(\hat{X}_{t}\right)^{-k}$ is a submartingale in $\mathscr{D}$. Since

$$
\left\{\begin{array}{l}
\left.h^{-k}\right|_{x \in \partial B_{R}} \leq(2-1+0)^{-k}=1 \\
\left.h^{-k}\right|_{x \in \partial B_{\beta R}} \leq\left(2-\beta^{2}\right)^{-k} \\
\left.h^{-k}\right|_{t=\theta R^{2}} \leq(2-1+1)^{-k} \leq\left(2-\beta^{2}\right)^{-k}
\end{array},\right.
$$

by the optional stopping theorem, we have for $x \in B_{R} \backslash \bar{B}_{\beta R}$,

$$
u(x, 0)+\left(2-\beta^{2}\right)^{k} h(x, 0)^{-k} \leq \sup _{\partial^{\mathrm{P}} \mathscr{D}}\left[u+\left(2-\beta^{2}\right)^{k} h^{-k}\right] \leq\left(2-\beta^{2}\right)^{k} .
$$

Therefore, for any $x \in B_{R} \backslash \bar{B}_{\beta R}$,

$$
\begin{aligned}
u(x, 0) & \leq\left(2-\beta^{2}\right)^{k}\left(1-h(x, 0)^{-k}\right) \\
& \leq C(h(x, 0)-1)=C\left[1-|x|^{2} /(R+1)^{2}\right] \\
& \leq C \operatorname{dist}\left(x, \partial B_{R}\right) / R .
\end{aligned}
$$

Our proof of Lemma 18 is complete.

\section{A local volume-doubling property and its consequences}

The purpose of this section is to obtain the parabolic VDP (Theorem 8) and a negative moment estimate (Theorem 26) for the density $\rho_{\omega}$. The former is an essential part for the proof of the PHI for $\mathcal{L}_{\omega}^{*}$, while the latter will imply the negative moment bound (8) for the heat kernel. Their proofs rely crucially on a VDP for hitting probabilities restricted in a finite ball (Lemma 19), which is an improved version of [28, Theorem 1.1] by Safonov and Yuan in the PDE setting.

As a by-product, we obtain a new proof of the classical PHI of Krylov and Safonov [22] in the lattice (Proposition 5). Our proof can be viewed as the parabolic version of Fabes and Stroock's [17] proof of the elliptic HI in the static PDE setting.

In the course of our proof, we also use the maximum principle (Theorem A.3.1) and mean-value inequality (Theorem A.4.1) both of which are standard results for $\omega$-caloric functions. Their statements and proofs are included in Sects. A.3 and A.4 for completeness. 


\subsection{Volume-doubling properties}

By the optional stopping theorem, for any $(x, t) \in \mathscr{D} \subset \mathbb{Z}^{d} \times \mathbb{R}$ and any bounded integrable function $u$ on $\mathscr{D} \cup \partial^{\mathrm{P}} \mathscr{D}$,

$$
u(x, t)=-E_{\omega}^{x, t}\left[\int_{0}^{\tau} \mathcal{L}_{\omega} u\left(\hat{X}_{r}\right) \mathrm{d} r\right]+E_{\omega}^{x, t}\left[u\left(\hat{X}_{\tau}\right)\right]
$$

where $\tau=\inf \left\{r \geq 0:\left(X_{r}, T_{r}\right) \notin \mathscr{D}\right\}$.

To prove Theorem 8, a crucial estimate is a VDP (Lemma 19) for the hitting measure of the random walk, which we will obtain by adapting some ideas of Safonov and Yuan [28] in the PDE setting. In contrast to [28, Theorem 1.1], our proof relies on a probabilistic estimate (Lemma 15) rather than the PHI (Proposition 5).

Lemma 19 Assume $\omega \in \Omega_{\kappa}$. Recall $\Delta(A, s)$ in (11). There exists $k_{0}=k_{0}(d, \kappa)$ such that for any $k \geq k_{0}, m \geq 2, r, s>0$ and $y \in B_{k \sqrt{s}}$, we have

$$
P_{\omega}^{y, 0}\left(X_{\Delta\left(B_{m k \sqrt{s}}, s\right)} \in B_{2 r}\right) \leq C_{k} P_{\omega}^{y, 0}\left(X_{\Delta\left(B_{m k \sqrt{s}}, s\right)} \in B_{r}\right)
$$

Here $C_{k}$ depends only on $(k, d, \kappa)$. In particular, for any $k \geq 1,|y| \leq k \sqrt{s}$,

$$
P_{\omega}^{y, 0}\left(X_{s} \in B_{2 r}\right) \leq C_{k} P_{\omega}^{y, 0}\left(X_{s} \in B_{r}\right) .
$$

Proof of Lemma 19: Since $B_{1}=\{0\}$, we only consider $r \geq 1 / 2$. Fix $s, r$, and let $k_{0} \geq 1$ be a large constant to be determined. For $\rho \geq 0, k \geq k_{0}$, define $L_{k, \rho}=B_{k \rho} \times\left\{s-\rho^{2}\right\}$ and (See Fig. 3.)

$$
D_{k, \rho}=\bigcup_{R \leq \rho} L_{k, R}=\left\{(x, t) \in \mathbb{Z}^{d} \times(-\infty, s]:|x| / k \leq \sqrt{s-t} \leq \rho\right\}
$$

For any $R \leq \rho$, by Lemma 15 , there exists $\alpha_{k}>0$ depending on $(k, \kappa, d)$ such that

$$
\begin{aligned}
\min _{\hat{x} \in L_{k, R}} P_{\omega}^{\hat{x}}\left(X_{\Delta\left(B_{m k \rho}, s\right)} \in B_{r}\right) & \geq \min _{x \in B_{k R}} P_{\omega}^{x, s-R^{2}}\left(X_{\Delta\left(B_{2 k R}, s\right)} \in B_{r}\right) \\
& \geq\left(\frac{r}{2 k R} \wedge \frac{1}{2}\right)^{\alpha_{k}}
\end{aligned}
$$

Let $\beta_{k}>1$ be a large constant to be determined later. Then, letting

$$
v_{\rho}(\hat{x})=(8 k)^{\alpha_{k}}\left(\beta_{k}+1\right) P_{\omega}^{\hat{x}}\left(X_{\Delta\left(B_{m k \rho}, s\right)} \in B_{r}\right)-P_{\omega}^{\hat{x}}\left(X_{\Delta\left(B_{m k \rho}, s\right)} \in B_{2 r}\right),
$$

we get $\inf _{D_{k, R}} v_{\rho} \geq\left(\beta_{k}+1\right)\left(\frac{4 r}{R} \wedge 2 k\right)^{\alpha_{k}}-1$ for $0<R \leq \rho$. In particular, $\inf _{D_{k,(4 r) \wedge \rho}} v_{\rho} \geq \beta_{k}$ for $\rho \geq 0$. Set

$$
R_{\rho}=\sup \left\{R \in[0, m \rho]: \inf _{D_{k, R}} v_{\rho} \geq 0\right\}
$$


Fig. 3 The shaded region is $D_{k, \rho}$

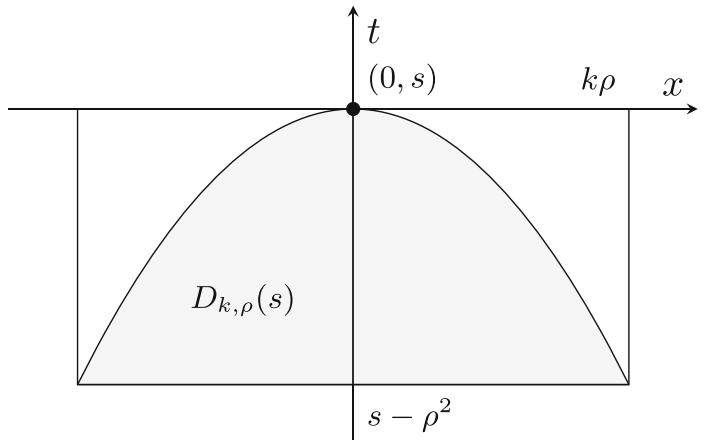

Clearly, $R_{\rho} \geq(4 r) \wedge \rho$. We will prove that

$$
R_{\rho} \geq \rho \text { for all } \rho>0 \text {. }
$$

Assuming (15) fails, then $R_{\rho}<\rho$ for some $\rho>4 r$. We will show that this is impossible via contradiction. First, for such $\rho>4 r$, we claim that there exists a constant $\gamma=\gamma(d, \kappa)>0$ such that

$$
\min _{L_{1, R}} v_{\rho} \geq \beta_{k}\left(\frac{r}{R}\right)^{\gamma} \quad \text { for all } R \in\left[2 r, R_{\rho}\right)
$$

By Lemma 15, $g(R):=\min _{x \in B_{R}} P_{\omega}^{x, s-R^{2}}\left(X_{\Delta\left(B_{2 R}, s-(R / 2)^{2}\right)} \in B_{R / 2}\right) \geq C$. Further, by the Markov property and that $R_{\rho}<\rho$, for $R \in\left[2 r, R_{\rho}\right), n \geq 1$,

$$
\min _{x \in B_{R}} P_{\omega}^{x, s-R^{2}}\left(X_{\Delta\left(D_{k, m \rho}, s-\left(R / 2^{n}\right)^{2}\right)} \in B_{R / 2^{n}}\right) \geq g(R) \cdots g\left(R / 2^{n-1}\right) \geq C^{n},
$$

Since $v_{\rho}\left(\hat{X}_{t}\right)$ is a martingale in $B_{m k \rho} \times(-\infty, s)$ and that $v_{\rho} \geq 0$ in $D_{k, R_{\rho}}$, choosing $n$ such that $R / 2^{n} \leq r<R / 2^{n-1}$, the above inequality yields

$$
\begin{aligned}
v_{\rho}\left(x, s-R^{2}\right) & \geq P_{\omega}^{x, s-R^{2}}\left(X_{\Delta\left(D_{k, m \rho}, s-\left(R / 2^{n}\right)^{2}\right)} \in B_{\left.R / 2^{n}\right)} \inf _{D_{k, r}} v_{\rho}\right. \\
& \geq C^{n} \beta_{k} \geq \beta_{k}\left(\frac{r}{R}\right)^{c}
\end{aligned}
$$

for $R \in\left[2 r, R_{\rho}\right.$ ) and $x \in B_{R}$. Display (16) is proved.

Next, we will show that for $R \in\left[2 r, R_{\rho}\right)$,

$$
f_{\rho}(R):=\sup _{\partial B_{k R} \times\left[s-R^{2}, s\right)} v_{\rho}^{-} \leq\left(\frac{r}{R}\right)^{-c / \log q_{k}},
$$

where $v_{\rho}^{-}=\max \left\{0,-v_{\rho}\right\}, q_{k}=1-\frac{c_{1}}{k}$ and $c_{1}>0$ is a constant to be determined. Noting that $v_{\rho}^{-}=0$ in $D_{k, R_{\rho}} \cup\left(B_{2 r}^{c} \times\{s\}\right)$ and tshat $v_{\rho}^{-}\left(\hat{X}_{t}\right)$ is a sub-martingale, we know that $f_{\rho}(R)$ is a decreasing function for $R \in\left(\frac{2 r}{k}, R_{\rho}\right)$. Further, for any 
$(x, t) \in \partial B_{k R} \times\left[s-R^{2}, s\right)$ with $q_{k} R \in\left(\frac{2 r}{k}, R_{\rho}\right)$, by the optional stopping lemma, in view of Lemma 13 ,

$$
\begin{aligned}
v_{\rho}^{-}(x, t) & \leq P_{\omega}^{x, t}\left(X_{a} \in \partial B_{k q_{k} R} \text { for some } a \in\left[0, R^{2}\right]\right) f\left(q_{k} R\right) \\
& \leq P_{\omega}^{x, t}\left(\sup _{0 \leq a \leq R^{2}}\left|X_{a}-x\right| \geq\left(1-q_{k}\right) k R\right) f\left(q_{k} R\right) \\
& \stackrel{\operatorname{Lemma} 13}{\leq} C\left(e^{-c c_{1}^{2}}+e^{-c_{1} R}\right) f\left(q_{k} R\right) \leq f\left(q_{k} R\right) / 2,
\end{aligned}
$$

if $c_{1}$ is chosen to be big enough. So $f_{\rho}(R) \leq f_{\rho}\left(q_{k} R\right) / 2$. Let $n \geq 0$ be the integer such that $q_{k}^{n+1} R<r \leq q_{k}^{n} R$. We conclude that for $R \in\left[2 r, R_{\rho}\right)$,

$$
f_{\rho}(R) \leq 2^{-n} f_{\rho}\left(q_{k}^{n} R\right) \leq\left(\frac{r}{R}\right)^{-c / \log q_{k}} f_{\rho}(r)
$$

Inequality (17) then follows from the fact $v_{\rho}^{-} \leq 1$.

Finally, if $R_{\rho}<\rho$ for some $\rho>4 r$, let $\tau=\inf \left\{t \geq 0: \hat{X}_{t} \notin\left(B_{m k \rho} \times(-\infty, s)\right) \backslash\right.$ $\left.\left(\bar{B}_{k R / 2} \times\left[s-(R / 2)^{2}, s\right)\right)\right\}$. Since $v_{\rho}=0$ on $\partial^{\mathrm{P}}\left(B_{m k \rho} \times(-\infty, s)\right) \backslash\left(B_{2 r} \times\{s\}\right)$, by the optional stopping lemma, for $R \in\left[R_{\rho}, 2 R_{\rho}\right)$ and $x \in B_{k R}$,

$$
\begin{aligned}
& v_{\rho}\left(x, s-R^{2}\right) \\
& =E_{\omega}^{x, s-R^{2}}\left[v_{\rho}\left(\hat{X}_{\tau}\right) \mathbb{1}_{\hat{X}_{\tau} \in B_{k R / 2} \times\left\{s-(R / 2)^{2}\right\} \text { or } \hat{X}_{\tau} \in \partial B_{k R / 2} \times\left[s-(R / 2)^{2}, s\right)}\right] \\
& \geq P_{\omega}^{x, s-R^{2}}\left(X_{\Delta\left(B_{2 k \rho}, s-(R / 2)^{2}\right)} \in B_{R / 2}\right) \min _{L_{1, R / 2}} v_{\rho}-f_{\rho}(R / 2) \\
& \stackrel{\text { Lemma }}{\longrightarrow}{ }^{15,(16),(17)} A_{k} \beta_{k}\left(\frac{2 r}{R}\right)^{\gamma}-\left(\frac{2 r}{R}\right)^{-c / \log q_{k}},
\end{aligned}
$$

where $A_{k}$ depends on $(k, \kappa, d)$. Taking $k_{0}>c_{1}$ to be big enough such that $-c / \log q_{k}>\gamma$ for $k \geq k_{0}$ and choosing $\beta_{k}>A_{k}^{-1}$, the above inequality then implies $\inf _{D_{k, 2 R_{\rho}}} v \geq 0$, which contradicts our definition of $R_{\rho}$. Display (15) is proved, and therefore, $\min _{x \in B_{k \sqrt{s}}} v_{\sqrt{s}}(x, 0) \geq 0$. The lemma follows.

Corollary 20 Let $\omega \in \Omega_{\kappa}$ and $k_{0}$ as in Lemma 19. For any $r>0, k \geq k_{0}, m \geq 2, s>0$ and $y \in B_{k \sqrt{s}}$, we have

$$
\sup _{t \geq 0:|t-s| \leq r^{2}} P_{\omega}^{y, 0}\left(X_{\Delta\left(B_{m k \sqrt{s}}, t\right)} \in B_{2 r}\right) \leq C_{k} P_{\omega}^{y, 0}\left(X_{\Delta\left(B_{m k \sqrt{s}}, s\right)} \in B_{r}\right),
$$

where $C_{k}$ depends on $(k, \kappa, d)$. In particular, for any $k \geq 1,|y| \leq k \sqrt{s}$,

$$
\sup _{t \geq 0:|t-s| \leq r^{2}} P_{\omega}^{y, 0}\left(X_{t} \in B_{2 r}\right) \leq C_{k} P_{\omega}^{y, 0}\left(X_{s} \in B_{r}\right)
$$


Proof It suffices to consider $r<\sqrt{s}$, because otherwise, by Lemma 15, the right side is bigger than a constant. When $t \in\left[0 \vee\left(s-r^{2}\right), s\right]$,

$$
\begin{aligned}
& \min _{y \in B_{k \sqrt{s}}} P_{\omega}^{y, 0}\left(X_{\Delta\left(B_{m k \sqrt{s}}, s\right)} \in B_{4 r}\right) \\
& \geq \min _{y \in B_{k \sqrt{s}}} P_{\omega}^{y, 0}\left(X_{\Delta\left(B_{m k \sqrt{s}}, t\right)} \in B_{2 r}\right) \min _{x \in B_{2 r}} P_{\omega}^{x, t}\left(X_{s-t} \in B_{2 r}(x)\right) \\
& \stackrel{\text { Lemma } 15}{\geq}{ }^{\geq} \min _{y \in B_{k \sqrt{s}}} P_{\omega}^{y, 0}\left(X_{\Delta\left(B_{m k \sqrt{s}}, t\right)} \in B_{2 r}\right) .
\end{aligned}
$$

By Lemma 19, we can replace $4 r$ in the above inequality by $r$.

When $t \in\left[s, s+r^{2}\right]$, for any $y \in B_{k \sqrt{s}}$,

$$
\begin{aligned}
& P_{\omega}^{y, 0}\left(X_{\Delta\left(B_{m k \sqrt{s}}, t\right)} \in B_{2 r}\right) \\
& \leq \sum_{n=0}^{\infty} \sum_{x:|x| \in\left[2^{n} r, 2^{n+1} r\right)} P_{\omega}^{y, 0}\left(X_{\Delta\left(B_{m k \sqrt{s}}, s\right)}=x\right) P_{\omega}^{x, s}\left(X_{t-s} \in B_{2 r}\right) \\
& \stackrel{\text { Corollary } 14}{\leq} C \sum_{n=0}^{\infty} P_{\omega}^{y, 0}\left(X_{\Delta\left(B_{m k \sqrt{s}}, s\right)} \in B_{2^{n} r}\right)\left(e^{-c 2^{n} r}+e^{-c 4^{n}}\right) .
\end{aligned}
$$

Observing that (cf. Lemma 19)

$$
P_{\omega}^{y, 0}\left(X_{\Delta\left(B_{m k \sqrt{s}}, s\right)} \in B_{2^{n} r}\right) \leq C^{n} P_{\omega}^{y, 0}\left(X_{\Delta\left(B_{m k \sqrt{s}}, s\right)} \in B_{r}\right)
$$

our proof is complete.

We define, for $\hat{x}=(x, t) \in \mathbb{R}^{d} \times \mathbb{R}$, the parabolic balls

$$
Q_{r}(\hat{x})=B_{r}(x) \times\left[t, t+r^{2}\right), \quad Q_{r}=Q_{r}(\hat{0}) .
$$

Proof of Theorem 8: Let $k_{0} \geq 2$ be as in Lemma 19. Recall $\bar{\omega}_{t}, \Delta, Q_{r}$ in (1), (11), (18). For fixed $\xi \in \Omega_{\kappa}$, define a probability measure $\mathbb{Q}_{R}=\mathbb{Q}_{R}^{\xi}$ on $\left\{\theta_{\hat{x}} \xi: \hat{x} \in Q_{R}\right\}$ such that for any bounded measurable $f \in \mathbb{R}^{\Omega}$,

$$
E_{\mathbb{Q}_{R}}[f]=\frac{1}{C_{R}} E_{\xi}^{0,-R^{2}}\left[\int_{0}^{\Delta\left(B_{2 k_{0} R}, R^{2}\right)} f\left(\bar{\xi}_{s}\right) \mathbb{1}_{\hat{X}_{s} \in Q_{R}} \mathrm{~d} s\right],
$$

where $C_{R}$ is a normalization constant such that $\mathbb{Q}_{R}$ is a probability. 
First, we claim that $C_{R} \asymp R^{2}$. Clearly, $C_{R} \leq 2 R^{2}$. On the other hand,

$$
\begin{aligned}
C_{R} & =E_{\xi}^{0,-R^{2}}\left[\int_{0}^{\Delta\left(B_{2 k_{0} R}, R^{2}\right)} \mathbb{1}_{\hat{X}_{s} \in Q_{R}} \mathrm{~d} s\right] \\
& \geq P_{\xi}^{0,-R^{2}}\left(X_{\Delta\left(B_{R}, 0\right)} \in B_{R / 2}\right) \min _{x \in B_{R / 2}} E_{\xi}^{x, 0}\left[\Delta\left(B_{R}, R^{2}\right)\right] \\
& \stackrel{\operatorname{Lemma} 15}{ }{ }^{\geq} \min _{x \in B_{R / 2}} E_{\xi}^{x, 0}\left[\Delta\left(B_{R}, R^{2}\right)\right] .
\end{aligned}
$$

Since $\left|X_{t}-X_{0}\right|^{2}-\frac{d}{\kappa} t$ is a supermartingale, denoting $\tau=\Delta\left(B_{R}, R^{2}\right)$, we have $0 \geq E_{\xi}^{x, 0}\left[\left|X_{\tau}-x\right|^{2}-\frac{d}{\kappa} \tau\right]$. Hence for any $x \in B_{R / 2}$,

$$
E_{\xi}^{x, 0}[\tau] \geq c E_{\xi}^{x, 0}\left[\left|X_{\tau}-x\right|^{2}\right] \geq C R^{2} P_{\xi}^{x, 0}\left(\tau<R^{2}\right),
$$

which implies $E_{\xi}^{x, 0}[\tau] \geq c R^{2}$. Thus $C_{R} \geq C R^{2}$ and so $C_{R} \asymp R^{2}$.

Next, since $\Omega$ is pre-compact, by Prohorov's theorem, there is a subsequence of $\mathbb{Q}_{R}$ that converges weakly, as $R \rightarrow \infty$, to a probability measure $\tilde{\mathbb{Q}}$ on $\Omega$. We will show that $\tilde{\mathbb{Q}}$ is an invariant measure of the process $\left(\bar{\omega}_{t}\right)$. Indeed, let $p_{R}=p_{R, \xi}$ denote the kernel $p_{R}(\hat{x} ; y, s):=P_{\xi}^{\hat{x}}\left(\hat{X}_{\Delta\left(B_{2 k_{0} R}, s\right)}=(y, s)\right)$. Then, letting $\mathscr{L} f(\omega)=$ $\sum_{e} \omega_{0}(0, e)\left[f\left(\theta_{e, 0} \omega\right)-f(\omega)\right]+\left.\partial_{t} f\left(\theta_{0, t} \omega\right)\right|_{t=0}$ denote the generator of the process $\left(\bar{\omega}_{t}\right)$, and $\hat{y}:=(y, s)$, we have

$$
E_{\mathbb{Q}_{R}}[\mathscr{L} f(\omega)]=C_{R}^{-1} \sum_{y \in B_{R}} \int_{0}^{R^{2}} p_{R}\left(0,-R^{2} ; \hat{y}\right) \mathscr{L} f\left(\theta_{\hat{y}} \xi\right) \mathrm{d} s
$$

for $f \in \operatorname{dom}(\mathscr{L})$, where $\operatorname{dom}(\mathscr{L})$ denotes the domain of the generator $\mathscr{L}$. Note that similar to $\rho_{\omega}$, the function $v(\hat{x})=p_{R}\left(0,-R^{2} ; \hat{x}\right)$ satisfies the equality $(3): \mathscr{L}^{T} v(\hat{x})=$ 0 for $\hat{x} \in B_{2 R} \times\left(-R^{2}, R^{2}\right)$, where $\mathscr{L}^{T} v(\hat{x})=\sum_{y} v(y, t) \omega_{t}(y, x)-\partial_{t} v(x, t)$. Hence, using integration by parts,

$$
\begin{aligned}
& \left|\sum_{y \in B_{R}} \int_{0}^{R^{2}} p_{R}\left(0,-R^{2} ; \hat{y}\right) \mathscr{L} f\left(\theta_{\hat{y}} \xi\right) \mathrm{d} s\right| \\
& \leq C\|f\|_{\infty} \int_{0}^{R^{2}} \sum_{y \in \bar{B}_{R} \backslash \stackrel{\leftrightarrow}{B}_{R}} p_{R}\left(0,-R^{2} ; \hat{y}\right) \mathrm{d} s+2\|f\|_{\infty}
\end{aligned}
$$

for all $f \in \operatorname{dom}(\mathscr{L})$, where $\stackrel{\circ}{B}_{R}=\left\{x \in B_{R}: x \nsim \partial B_{R}\right\}$. Observe that

$$
u(\hat{x})=\int_{0}^{R^{2}} \sum_{y \in \bar{B}_{R} \backslash \stackrel{\circ}{B}_{R}} p_{R}(\hat{x} ; \hat{y}) \mathrm{d} s=E_{\xi}^{\hat{x}}\left[\int_{0}^{\Delta\left(B_{2 k_{0} R}, R^{2}\right)} \mathbb{1}_{\hat{X}_{t} \in \bar{B}_{R} \backslash \stackrel{\circ}{B}_{R} \times\left(0, R^{2}\right)}\right] \mathrm{d} t
$$


satisfies $\mathcal{L}_{\xi} u(\hat{x})=-\mathbb{1}_{\hat{x} \in \bar{B}_{R} \backslash \stackrel{\circ}{B}_{R} \times\left[0, R^{2}\right)}$ for $\hat{x} \in \mathscr{D}:=B_{2 k_{0} R} \times\left[-R^{2}, R^{2}\right)$ and $\left.u\right|_{{ }^{\mathrm{P}} \mathscr{D}}=$ 0 . By the parabolic maximum principle (Theorem A.3.1), we get $u\left(0,-R^{2}\right) \leq$ $C R^{(2 d+1) /(d+1)}$. Hence, by (19), (20), and $C_{R} \asymp R^{2}$,

$$
\lim _{R \rightarrow \infty} E_{\mathbb{Q}_{R}}[\mathscr{L} f]=0 \quad \forall \text { bounded function } f \in \operatorname{dom}(\mathscr{L})
$$

and so $E_{\tilde{\mathbb{Q}}}[\mathscr{L} f]=0$, which implies that $\tilde{\mathbb{Q}}$ is an invariant measure of $\left(\bar{\omega}_{t}\right)$.

Furthermore, we will show that $\tilde{\mathbb{Q}} \ll \mathbb{P}$. Notice that the function

$$
w(\hat{x}):=E_{\xi}^{\hat{x}}\left[\int_{0}^{\Delta\left(B_{2 k_{0} R}, R^{2}\right)} f\left(\bar{\xi}_{s}\right) \mathbb{1}_{\hat{X}_{s} \in Q_{R}} \mathrm{~d} s\right]
$$

satisfies $\mathcal{L}_{\xi} w(\hat{x})=-f\left(\theta_{\hat{x}} \xi\right) \mathbb{1}_{\hat{x} \in Q_{R}}$ in $\mathscr{D}$ and $\left.w\right|_{\partial^{\mathrm{P}} \mathscr{D}}=0$. By Theorem A.3.1, for any bounded measurable $f \in \mathbb{R}^{\Omega}$,

$$
E_{\mathbb{Q}_{R}}[f] \leq C R^{-2} w\left(0,-R^{2}\right) \leq C\left[\int_{0}^{R^{2}} \sum_{x \in B_{R}}\left|f\left(\theta_{x, t} \xi\right)\right|^{d+1} \mathrm{~d} t /\left(R^{2+d}\right)\right]^{1 /(d+1)},
$$

which, by the multi-dimensional ergodic theorem, yields $E_{\tilde{\mathbb{Q}}}[f] \leq C\|f\|_{L^{d+1}(\mathbb{P})}$ as we take $R \rightarrow \infty$. So $\tilde{\mathbb{Q}} \ll \mathbb{P}$. By Theorem $1, \tilde{\mathbb{Q}}=\mathbb{Q}$.

Finally, since $\mathbb{Q}_{R} \Rightarrow \mathbb{Q}$, for any bounded measurable $f \in \mathbb{R}^{\Omega}$,

$$
\begin{aligned}
& E_{\mathbb{P}}\left[\rho_{\omega}\left(B_{r}, t\right) f\right]=\sum_{x \in B_{r}} E_{\mathbb{Q}}\left[f\left(\theta_{x,-t} \omega\right)\right] \\
& =\lim _{R \rightarrow \infty} \sum_{x \in B_{r}, y \in B_{R}} \int_{0}^{R^{2}} P_{\xi}^{0,-R^{2}}\left(X_{\Delta\left(B_{2 k_{0} R}, s\right)}=y\right) f\left(\theta_{x+y, s-t} \xi\right) \mathrm{d} s / C_{R} .
\end{aligned}
$$

Hence, for any measurable function $f \geq 0,|t| \leq r^{2}$, and $\mathbb{P}$-a.a. $\xi$,

$$
\begin{aligned}
& E_{\mathbb{P}}\left[\rho_{\omega}\left(B_{r}, 0\right) f\right] \\
& \geq \lim _{R \rightarrow \infty} \sum_{z \in B_{R-r}} \int_{0}^{R^{2}} P_{\xi}^{0,-R^{2}}\left(X_{\Delta\left(B_{2 k_{0} R}, s\right)} \in B_{r}(z)\right) f\left(\theta_{z, s} \xi\right) \mathrm{d} s / C_{R} \\
& \stackrel{\text { Corollary } 20}{\geq} \lim _{R \rightarrow \infty} \sum_{z \in B_{R-r}} \int_{0}^{R^{2}} P_{\xi}^{0,-R^{2}}\left(X_{\Delta\left(B_{2 k_{0} R}, s+t\right)} \in B_{2 r}(z)\right) f\left(\theta_{z, s} \xi\right) \mathrm{d} s / C_{R} \\
& \geq C \lim _{R \rightarrow \infty} \sum_{x \in B_{2 r}, y \in B_{R-3 r}} \int_{r^{2}}^{R^{2}-r^{2}} P_{\xi}^{0,-R^{2}}\left(X_{\Delta\left(B_{2 k_{0} R}, s\right)}=y\right) f\left(\theta_{x+y, s-t} \xi\right) \mathrm{d} s / C_{R} \\
& \stackrel{(21)}{=} C E_{\mathbb{P}}\left[\rho_{\omega}\left(B_{2 r}, t\right) f\right] .
\end{aligned}
$$

Since $f$ is arbitrary, the theorem follows. 
Remark 21 By Theorem 8, for any $r \geq 1$,

$$
\frac{c}{r^{2}} \int_{0}^{r^{2}} \rho_{\omega}\left(B_{r}, s\right) \mathrm{d} s \leq \rho_{\omega}\left(B_{r}, 0\right) \leq \frac{C}{r^{2}} \int_{0}^{r^{2}} \rho_{\omega}\left(B_{r}, s\right) \mathrm{d} s .
$$

Hence, by the multi-dimensional ergodic theorem, for $\mathbb{P}$-almost every $\omega$,

$$
c \leq \lim _{r \rightarrow \infty} \frac{1}{\left|B_{r}\right|} \rho_{\omega}\left(B_{r}, 0\right) \leq \varlimsup_{r \rightarrow \infty} \frac{1}{\left|B_{r}\right|} \rho_{\omega}\left(B_{r}, 0\right) \leq C .
$$

Display (23) will be used in the Proof of Theorem 4 in Sect. 6.

\section{2 $A_{p}$ property and proof of the PHI for $\mathcal{L}_{\omega}$}

The goal of this subsection is to obtain a negative moment bound for the density $\rho_{\omega}$ and to prove the PHI for $\mathcal{L}_{\omega}$. We will first obtain a reverse Hölder inequality for adjoint solutions, and then use it to imply the negative moment bounds and the PHI.

We endow $\mathbb{Z}^{d}$ with the discrete topology and counting measure, and equip $\mathbb{Z}^{d} \times \mathbb{R}$ with the corresponding product topology and measure (where $\mathbb{R}$ has the usual topology and measure). For $\mathscr{D} \subset \mathbb{Z}^{d} \times \mathbb{R}$, let $|\mathscr{D}|$ be its measure, and denote the integration over $\mathscr{D}$ by $\int_{\mathscr{D}} f$. For instance,

$$
\int_{B_{R} \times[0, T]} f=\sum_{x \in B_{R}} \int_{0}^{T} f(x, t) \mathrm{d} t
$$

and $|\mathscr{D}|=\int_{\mathscr{D}} 1$. For $p>0$, we define a norm

$$
\|f\|_{\mathscr{D}, p}:=\left(\int_{\mathscr{D}}|f|^{p} /|\mathscr{D}|\right)^{1 / p}
$$

We write $f(\mathscr{D}):=\int_{\mathscr{D}} f$.

Definition 22 A function $v \in \mathbb{R}^{\mathbb{Z}^{d} \times \mathbb{R}}$ is called an adjoint solution of $\mathcal{L}_{\omega}$ in $\mathscr{D}=$ $B_{R} \times\left[T_{1}, T_{2}\right)$ if $\int_{\mathscr{D}} v \mathcal{L}_{\omega} \phi=0$ for any test function $\phi \overline{(x, t) \in \mathbb{R}^{\mathbb{Z}^{d} \times \mathbb{R}}}$ that is supported on $B_{R} \times\left(T_{1}, T_{2}\right)$ and smooth in $t$.

For $\hat{x}=\left(x_{1}, \ldots, x_{d}, t\right)$, define parabolic cubes with side-length $r>0$ as

$$
K_{r}(\hat{x})=\left(\prod_{i=1}^{d}\left[x_{i}-r, x_{i}+r\right) \cap \mathbb{Z}^{d}\right) \times\left[t, t+r^{2}\right), \quad K_{r}=K_{r}(\hat{0}) .
$$

We say that a function $w \in \mathbb{R}^{\mathbb{Z}^{d} \times \mathbb{R}}$ satisfies the reverse Hölder inequality $R H_{q}(\mathscr{D})$, $1<q<\infty$, if for any parabolic subcube $K$ of $\mathscr{D}$,

$$
\|w\|_{K, q} \leq C\|w\|_{K, 1}
$$


We say that $w$ belongs to the $A_{p}(\mathscr{D})$ class (with $A_{p}$ bound $A$ ), $1<p<\infty$, if there exists $A<\infty$ such that, for any parabolic subcube $K$ of $\mathscr{D}$,

$$
\|w\|_{K, 1}\|1 / w\|_{K, 1 /(p-1)} \leq A
$$

The following lemma is useful in the derivation of reverse Hölder inequalities for adjoint solutions.

Lemma 23 Recall $\|\cdot\|_{\mathscr{D}, p}$ in (25) and the parabolic balls $Q_{r}$ in (18). Let $\omega \in \Omega_{\kappa}$. For any non-negative adjoint solution $v$ of $\mathcal{L}_{\omega}$ in $Q_{2 r}, r>10$,

$$
\|v\|_{Q_{r},(d+1) / d} \leq C\|v\|_{Q_{3 r / 2}, 1} .
$$

Proof Denote the balls of radius $r$ by

$$
\mathcal{O}_{r}=\left\{x \in \mathbb{R}^{d}:|x|_{2}<r\right\} \quad \text { and } \quad \mathcal{O}_{r}(y)=y+\mathcal{O}_{r}, \quad y \in \mathbb{R}^{d} .
$$

Let $\phi_{0} \geq 0$ be a smooth (with respect to $t$ ) function supported on $\mathcal{O}_{3 / 2} \times[0,9 / 4$ ) with $\left.\phi_{0}\right|_{\mathcal{O}_{1} \times[0,1)}=1$ and set $\phi(x, t)=\phi_{0}\left(x / r, t / r^{2}\right)$. Let $f$ be any non-negative smooth function supported on $Q_{r}$ with $\|f\|_{Q_{r}, d+1}=1$ and let $u \in[0, \infty)^{\mathbb{Z}^{d} \times \mathbb{R}}$ be supported on $Q_{9 r / 5}$ with $L_{\omega} u=-f$ in $Q_{9 r / 5}$. Since

$0=\int v \mathcal{L}_{\omega}(\phi u)=\int v \phi \mathcal{L}_{\omega} u+\int v u \mathcal{L}_{\omega} \phi+\sum_{x, y} \int_{\mathbb{R}} v(x, t) \omega_{t}(x, y) \nabla_{x, y} u \nabla_{x, y} \phi \mathrm{d} t$

where $\nabla_{x, y} u(\cdot, t):=u(x, t)-u(y, t)$ and (cf. (24)) $\int=\int_{\mathbb{Z}^{d} \times \mathbb{R}}$, we get

$$
\int v \phi f=\int v u \mathcal{L}_{\omega} \phi+\sum_{x, y} \int_{\mathbb{R}} v(x, t) \omega_{t}(x, y) \nabla_{x, y} u \nabla_{x, y} \phi \mathrm{d} t=: \mathrm{I}+\mathrm{II} .
$$

By the maximum principle Theorem A.3.1, $u \leq C r^{2}\|f\|_{Q_{r}, d+1} \leq C r^{2}$. Thus, using $\left|\mathcal{L}_{\omega} \phi\right| \leq C / r^{2}$, we get $|\mathrm{I}| \leq C v\left(Q_{3 r / 2}\right)$. Further, noting that

$$
\begin{aligned}
\left|\sum_{x, y} \int_{\mathbb{R}} v(x, t) \omega_{t}(x, y)\left(\nabla_{x, y} u\right)^{2} \mathrm{~d} t\right| & =\left|\int v \mathcal{L}_{\omega}\left(u^{2}\right)-2 \int v u \mathcal{L}_{\omega} u\right| \\
& =2\left|\int v u \mathcal{L}_{\omega} u\right| \leq C r^{2} \int v f
\end{aligned}
$$


we have

$$
\begin{aligned}
|\mathrm{II}| & \leq\left(\sum_{x, y} \int_{\mathbb{R}} v(x, t) \omega_{t}(x, y)\left(\nabla_{x, y} \phi\right)^{2} \mathrm{~d} t\right)^{1 / 2}\left(\sum_{x, y} \int_{\mathbb{R}} v(x, t) \omega_{t}(x, y)\left(\nabla_{x, y} u\right)^{2} \mathrm{~d} t\right)^{1 / 2} \\
& \leq C v\left(Q_{3 r / 2}\right)^{1 / 2}\left(\int v f\right)^{1 / 2} .
\end{aligned}
$$

Hence we obtain $\int v f \leq \int v \phi f \leq C v\left(Q_{3 r / 2}\right)+C v\left(Q_{3 r / 2}\right)^{1 / 2}\left(\int v f\right)^{1 / 2}$ and so $v\left(Q_{3 r / 2}\right) \geq c \int v f$. The lemma follows by taking supremum over all $f$ with $\|f\|_{Q_{r}, d+1}=1$.

Recall the stopping time $\Delta$ in (11). For $R>0, \hat{y} \in B_{2 R} \times \mathbb{R}$, let

$$
g_{R}(\hat{y} ; x, t)=P_{\omega}^{\hat{y}}\left(X_{\Delta\left(B_{2 R}, t\right)}=x\right)
$$

Using Lemma 23, we obtain the following reverse Hölder inequalities for the functions $\rho_{\omega}(\cdot)$ and $g_{R}(\hat{y} ; \cdot)$.

Corollary 24 Let $\omega \in \Omega_{\kappa}, R>0$. Recall $k_{0}$ in Lemma 19.

(i) $\rho_{\omega}$ satisfies $R H_{(d+1) / d}\left(\mathbb{Z}^{d} \times \mathbb{R}\right)$.

(ii) For any $y \in B_{R}, v_{y}(\hat{x})=g_{R}(y, 0 ; \hat{x})$ satisfies

$$
R H_{(d+1) / d}\left(B_{R / 2} \times\left[R^{2} /\left(2 k_{0}^{2}\right), R^{2} / k_{0}^{2}\right]\right) .
$$

Proof Note that $\rho_{\omega}, v_{y}$ are adjoint solutions with volume-doubling properties Theorem 8 and Corollary 20. The corollary follows from Lemma 23.

As a classical result in harmonic analysis, reverse Hölder inequalities imply $\left(A_{p}\right)$. See e.g, [10, pg.246-249], [29, pg. 213-214]. We state this fact for our discrete setting as below, and include its proof in Sect. A.6.

Lemma 25 Let $K^{0} \subset \mathbb{Z}^{d} \times \mathbb{R}$ be a parabolic cube with side-length $r>0$. If a function $w>0$ on $K^{0}$ satisfies $R H_{q}\left(K^{0}\right), q>1$, then

(i) $w \in A_{p}\left(K^{0}\right)$ for some $1<p<\infty$;

(ii) $\frac{w(E)}{w(K)} \geq C\left(\frac{|E|}{|K|}\right)^{c}$ for all $E \subset K$ where $K \neq \emptyset$ is a subcube of $K^{0}$.

With Lemma 25 and the reverse Hölder inequalities for $\rho_{\omega}$ and $g_{R}(y, 0 ; \cdot)$, the following $A_{p}$ bounds and measure estimate follow immediately.

Theorem 26 Let $\omega, R, k_{0}, v_{y}$ be the same as in Corollary 24. There exist $p=$ $p(d, \kappa)>1, A=A(d, \kappa)$ such that, for $\mathbb{P}$-a.e. $\omega$,

(a) $\rho_{\omega} \in A_{p}\left(\mathbb{Z}^{d} \times \mathbb{R}\right)$ with $A_{p}$ bound $A$. As a consequence,

$$
E_{\mathbb{P}}\left[\rho_{\omega}^{-1 /(p-1)}\right]<\infty
$$


(b) For any $y \in B_{R}$, $v_{y}$ belongs to $A_{p}\left(B_{R / 2} \times\left[R^{2} /\left(2 k_{0}^{2}\right), R^{2} / k_{0}^{2}\right]\right)$ with $A_{p}$ bound $A$. Moreover, for any $E \subset K$ where $K$ is a parabolic subcube of $B_{R / 2} \times\left[R^{2} /\left(2 k_{0}^{2}\right), R^{2} / k_{0}^{2}\right]$,

$$
\frac{g_{R}(y, 0 ; E)}{g_{R}(y, 0 ; K)} \geq C\left(\frac{|E|}{|K|}\right)^{c}
$$

Remark 27 In the elliptic non-divergence form PDE setting, the $A_{p}$ inequality for adjoint solutions was proved by Bauman [6], and estimate of the form (30) was used by Fabes and Stroock [17] to obtain a short proof of the elliptic Harnack inequality.

Using (30), we will prove the PHI (Proposition 5). Our proof follows the ideas of [17].

Proof of Proposition 5 Let $\ell_{0}=1 / k_{0}^{2}$ and $\mathscr{D}=\left\{x:|x|_{\infty}<R / \sqrt{d}\right\} \times\left[\ell_{0} R^{2} / 2, \ell_{0} R^{2}\right]$. We only prove a weaker version $\sup _{\mathscr{D}} u \leq C \min _{x \in B_{R / \sqrt{4 d}}} u(x, 0)$. The PHI then follows by iteration. Indeed, assume $\min _{x \in B_{R / \sqrt{4 d}}} u(x, 0)=u(y, 0)=1$ for $y \in$ $B_{R / \sqrt{4 d}}$. Let $E_{\lambda}=\{\hat{x} \in \mathscr{D}: u(\hat{x}) \geq \lambda\}$. By Lemma 15, $g_{R}(y, 0 ; \mathscr{D})>C R^{2}$. Moreover, for $s \in\left[\ell_{0} R^{2} / 2, \ell_{0} R^{2}\right], 1=u(y, 0) \geq \lambda g_{R}\left(y, 0 ; E_{\lambda} \cap\{(x, t): t=s\}\right)$, and so

$$
1 \geq C \lambda g_{R}\left(y, 0 ; E_{\lambda}\right) / R^{2} \stackrel{\text { Theorem } 26(b)}{\geq} C \lambda\left(\left|E_{\lambda}\right| /|\mathscr{D}|\right)^{c} .
$$

Hence $\left|E_{\lambda}\right| /|\mathscr{D}| \leq C \lambda^{-\gamma}$ for some $\gamma>0$. Therefore, for $0<p<\gamma / 2$,

$$
\|u\|_{\mathscr{D}, p} \leq\left[1+p \int_{1}^{\infty} \lambda^{p-1}\left|E_{\lambda}\right| /|\mathscr{D}| \mathrm{d} \lambda\right]^{1 / p}<C^{\prime}=C^{\prime} \min _{x \in B_{R / \sqrt{d}}} u(x, 0)<\infty
$$

This inequality, together with the mean value inequality (Theorem A.4.1), completes our proof.

\section{Estimates of caloric functions near the boundary}

The purpose of this section is to establish estimates (Propositions 28 and 30) of $\omega$ caloric functions near the parabolic boundary. These estimates are important tools for our proof of the PHI for $\mathcal{L}_{\omega}^{*}$ in Sect. 5.

For $x \in \mathbb{Z}^{d}, A \subset \mathbb{Z}^{d}$, let

$$
\operatorname{dist}(x, A):=\min _{y \in A}|x-y|_{1} .
$$

\subsection{An elliptic-type Harnack inequality}

Proposition 28 (Interior elliptic-type Harnack inequality) Assume $\omega \in \Omega_{\kappa}, R \geq 2$. Suppose $u \geq 0$ is an $\omega$-caloric function on $Q_{R}$ with $u=0$ on $\partial B_{R} \times\left[0, R^{2}\right)$. Then 
Fig. 4 The values of $u$ are comparable inside the region $Q_{R}^{\delta}$

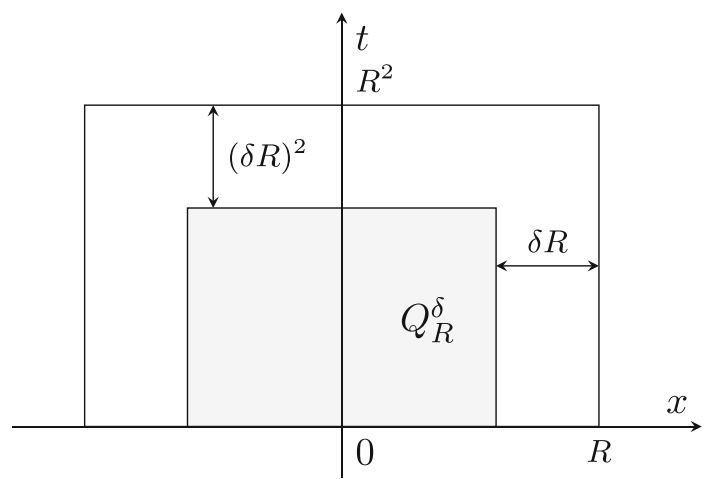

for $0<\delta \leq \frac{1}{4}$, letting $Q_{R}^{\delta}:=B_{(1-\delta) R} \times\left[0,\left(1-\delta^{2}\right) R^{2}\right)$, there exists a constant $C=C(d, \kappa, \delta)$ such that

$$
\sup _{Q_{R}^{\delta}} u \leq C \inf _{Q_{R}^{\delta}} u
$$

To prove Proposition 28, we need a so-called Carlson-type estimate. For parabolic differential operators in non-divergence form, this kind of estimate was first proved by Garofalo [18] (see also [16, Theorem 3.3]).

Lemma 29 (Carlson-type estimate) Assume $\omega \in \Omega_{\kappa}, R>2 r>0$. Suppose $u \geq 0$ is an $\omega$-caloric function on $\left(B_{R} \backslash \bar{B}_{R-2 r}\right) \times\left[0,3 r^{2}\right)$ with $u=0$ on $\partial B_{R} \times\left[r^{2}, 3 r^{2}\right)$. Then, with the convention $\sup \emptyset=-\infty$, we have

$$
\sup _{\left(B_{R} \backslash B_{R-r}\right) \times\left[r^{2}, 2 r^{2}\right)} u \leq C \min _{y \in \partial B_{R-r}} u(y, 0) .
$$

Proof Set $\mathscr{D}=\left(B_{R} \backslash \bar{B}_{R-2 r}\right) \times\left[r^{2}, 3 r^{2}\right)$. For $\hat{x}=(x, t) \in \mathscr{D}$, let $d_{1}(\hat{x})=\sup \{\rho \geq$ $\left.0: B_{\rho}(x) \subset B_{R} \backslash \bar{B}_{R-2 r}\right\} \geq 1$.

First, we show that there exists $\gamma=\gamma(d, \kappa)$ such that

$$
\sup _{\hat{x} \in \mathscr{D}}\left(d_{1}(\hat{x}) / r\right)^{\gamma} u(\hat{x}) \leq C \min _{y \in \partial B_{R-r}} u(y, 0) .
$$

Indeed, for any $\hat{x}=(x, t) \in \mathscr{D}$, we can find a sequence of $n \leq C \log \left(r / d_{1}(\hat{x})\right)$ balls with increasing radii $r_{k}:=c 2^{k} d_{1}(\hat{x})$ :

$$
B_{r_{1}}\left(x_{1}\right) \subset B_{r_{2}}\left(x_{2}\right) \subset \cdots \subset B_{r_{n}}\left(x_{n}\right) \subset B_{R} \backslash \bar{B}_{R-2 r}
$$

such that $x_{1}=x, \operatorname{dist}\left(x_{n}, \partial B_{R-r}\right) \leq r / 2$, and $t-r_{n}^{2} \geq r^{2} / 2$. By Proposition 5,

$$
\begin{aligned}
u(x, t) & \leq C u\left(x_{1}, t-r_{1}^{2}\right) \leq \cdots \\
& \leq C^{n} u\left(x_{n}, t-r_{n}^{2}\right) \leq C\left(\frac{r}{d_{1}(\hat{x})}\right)^{c} \min _{y \in \partial B_{R-r}} u(y, 0),
\end{aligned}
$$


where in the last inequality we applied Proposition 5 to a chain of parabolic balls with spatial centers at $\partial B_{R-r}$ and radius $c r$. Display (32) is proved.

Next, with $\gamma$ as in (32), letting $d_{0}(\hat{x})=\sup \left\{\rho \geq 0: Q_{\rho}(\hat{x}) \subset\left(\mathbb{Z}^{d} \backslash \bar{B}_{R-2 r}\right) \times\right.$ $\left.\left[r^{2}, 3 r^{2}\right)\right\}$, we claim that

$$
\sup _{\hat{x} \in \mathscr{D}} d_{0}(\hat{x})^{\gamma} u(\hat{x}) \leq \epsilon^{-\gamma} \sup _{\hat{y} \in \mathscr{D}} d_{1}(\hat{y})^{\gamma} u(\hat{y})
$$

where $\epsilon=\epsilon(d, \kappa) \in(0,1 / 5)$ is to be determined. It suffices to show that $\sup _{\mathscr{D}} d_{0}^{\gamma} u$ is achieved at $\hat{x} \in \mathscr{D}$ with $\epsilon d_{0}(\hat{x}) \leq d_{1}(\hat{x})$. Indeed, if $\epsilon d_{0}(\hat{x})>d_{1}(\hat{x})$, then $B_{2 d_{1}(\hat{x})} \backslash$ $B_{R} \neq \emptyset$, and for any $\hat{y}=(y, s) \in Q_{2 d_{1}(\hat{x})}(\hat{x}) \cap \mathscr{D}$,

$$
d_{0}(\hat{x}) \leq d_{0}(\hat{y})+|x-y|+|t-s|^{1 / 2} \leq d_{0}(\hat{y})+4 d_{1}(\hat{x}) \leq d_{0}(\hat{y})+4 \epsilon d_{0}(\hat{x})
$$

and so $d_{0}(\hat{x}) \leq(1-4 \epsilon)^{-1} d_{0}(\hat{y})$. Moreover, by Corollary 16 ,

$$
\begin{aligned}
d_{0}(\hat{x})^{\gamma} u(\hat{x}) \leq & {\left[1-P_{\omega}^{\hat{x}}\left(X . \text { exits } B_{2 d_{1}(\hat{x})}(x) \cap B_{R} \text { from } \partial B_{R} \text { before time } d_{1}^{2}(\hat{x})\right)\right] } \\
& \times d_{0}(\hat{x})^{\gamma} \sup _{\left(B_{2 d_{1}(\hat{x})}(x) \cap B_{R}\right) \times\left[r^{2}, 3 r^{2}\right)} u \\
\leq & \left(1-c_{0}\right)(1-4 \epsilon)^{-\gamma} \sup _{\mathscr{D}} d_{0}^{\gamma} u
\end{aligned}
$$

for a constant $c_{0} \in(0,1)$. Thus, when $\epsilon d_{0}(\hat{x})>d_{1}(\hat{x})$, choosing $\epsilon>0$ so that $\left(1-c_{0}\right)(1-4 \epsilon)^{-\gamma}<1-\frac{c_{0}}{2}$, we get $d_{0}(\hat{x})^{\gamma} u(\hat{x})<\left(1-\frac{c_{0}}{2}\right) \sup _{\mathscr{D}} d_{0}^{\gamma} u$. Display (33) is proved. Inequality (31) follows from (32) and (33).

Proof of Proposition 28: Since $u=0$ on $\partial B_{R} \times\left[0, R^{2}\right)$,

$$
\begin{aligned}
\sup _{Q_{R}^{\delta}} u & \leq \sup _{B_{R} \times\left\{R^{2}-\frac{1}{4}(\delta R)^{2}\right\}} u \\
& \leq C \quad \sup _{B_{(1-\delta) R} \times\left\{R^{2}-\frac{1}{2}(\delta R)^{2}\right\}} u \\
& \stackrel{\text { Proposition } 5}{\leq} C(d, \kappa, \delta) \inf _{Q_{R}^{\delta}} u,
\end{aligned}
$$

where we used Lemma 29 and Proposition 5 in the second inequality.

\subsection{A boundary Harnack inequality}

For positive caloric functions with zero values on the spatial boundary, the following boundary PHI compares values near the spatial boundary and values inside, with time coordinates appropriately shifted. 
Proposition 30 (Boundary PHI) Let $R>0$. Suppose $u$ is a nonnegative $\omega$-caloric function on $\left(B_{4 R} \backslash B_{2 R}\right) \times\left(-2 R^{2}, 3 R^{2}\right)$, and $\left.u\right|_{\partial B_{4 R} \times \mathbb{R}}=0$. Then for any $\hat{x}=$ $(x, t) \in\left(B_{4 R} \backslash \bar{B}_{3 R}\right) \times\left(-R^{2}, R^{2}\right)$, we have

$C \frac{\operatorname{dist}\left(x, \partial B_{4 R}\right)}{R} \max _{y \in \partial B_{3 R}} u\left(y, t+R^{2}\right) \leq u(\hat{x}) \leq C \frac{\operatorname{dist}\left(x, \partial B_{4 R}\right)}{R} \min _{y \in \partial B_{3 R}} u\left(y, t-R^{2}\right)$.

Proposition 30 is a lattice version of $[18,(3.9)]$. In what follows we offer a probabilistic proof.

Proof of Proposition 30: Our proof uses the fact that $u\left(\hat{X}_{t}\right)$ is a martingale before exiting the region $\mathscr{D}:=\left(B_{4 R} \backslash B_{2 R}\right) \times\left(-2 R^{2}, 3 R^{2}\right)$.

For the lower bound, let $\tau_{3,4}:=\inf \left\{s>0: X_{s} \notin B_{4 R} \backslash \bar{B}_{3 R}\right\}$. By the optional stopping lemma, $u(\hat{x})=E_{\omega}^{\hat{x}}\left[u\left(\hat{X}_{\tau_{3,4} \wedge 0.5 R^{2}}\right)\right]$, and so

$$
\begin{aligned}
u(\hat{x}) & \geq P_{\omega}^{x, t}\left(\tau_{3,4}<R^{2} / 2, X_{\tau_{3,4}} \in \partial B_{3 R}\right) \inf _{\partial B_{3 R} \times\left[t, t+0.5 R^{2}\right]} u \\
& \geq C \frac{\operatorname{dist}\left(x, \partial B_{4 R}\right)}{R} \max _{y \in \partial B_{3 R}} u\left(y, t+R^{2}\right)
\end{aligned}
$$

where in the last inequality we used Lemma 17 and applied Proposition 5 (to a chain of parabolic balls). The lower bound is obtained.

To obtain the upper bound, note that for $\hat{x} \in\left(B_{4 R} \backslash \bar{B}_{3 R}\right) \times\left(-R^{2}, R^{2}\right)$,

$$
\begin{aligned}
u(\hat{x}) & \leq\left[\max _{z \in B_{4 R} \backslash \bar{B}_{3 R}} u\left(z, t+\frac{R^{2}}{2}\right)+\underset{\partial B_{3 R} \times\left(t, t+\frac{R^{2}}{2}\right]}{\max } u\right] P_{\omega}^{x, t}\left(X_{\tau_{3,4} \wedge 0.5 R^{2}} \notin \partial B_{4 R}\right) \\
& \stackrel{(31)}{\leq} C\left[\max _{z \in B_{3.5 R} \backslash \bar{B}_{3 R}} u\left(z, t-\frac{R^{2}}{2}\right)+\underset{\partial B_{3 R} \times\left(t, t+\frac{R^{2}}{2}\right]}{\max } u\right] P_{\omega}^{x, t}\left(X_{\tau_{3,4} \wedge 0.5 R^{2}} \notin \partial B_{4 R}\right) \\
& \leq C \min _{z \in \partial B_{3 R}} u\left(z, t-R^{2}\right) \operatorname{dist}\left(x, \partial B_{4 R}\right) / R,
\end{aligned}
$$

where in the last inequality we applied Lemma 18 and used an iteration of the PHI for $\omega$-caloric functions (Proposition 5).

\section{Proof of the PHI for the adjoint operator (Theorem 6)}

In this section we will prove the PHI for $\mathcal{L}_{\omega}^{*}$. Our proof relies on a representation formula for $\omega^{*}$-caloric functions (Proposition 31), the parabolic volume-doubling property of $\rho_{\omega}$ (Theorem 8), the PHI (Proposition 5) and boundary PHI (Proposition 30) for $\omega$-caloric functions.

We define $\hat{Y}_{t}=\left(Y_{t}, S_{t}\right)$ to be the continuous-time Markov chain on $\mathbb{Z}^{d} \times \mathbb{R}$ with generator $\mathcal{L}_{\omega}^{*}$. The process $\hat{Y}_{t}$ can be interpreted as the time-reversal of $\hat{X}_{t}$. Denote by $P_{\omega^{*}}^{y, s}$ the quenched law of $\hat{Y}$. starting from $\hat{Y}_{0}=(y, s)$ and by $E_{\omega^{*}}^{y, s}$ the corresponding expectation. Note that $S_{t}=S_{0}-t$. 
For $R>0, \hat{x}=(x, t), \hat{y}=(y, s) \in B_{R} \times \mathbb{R}$ with $s>t$, set

$$
\begin{aligned}
& p_{R}^{\omega}(\hat{x} ; \hat{y})=P_{\omega}^{x, t}\left(X_{s-t}=y, s-t<\tau_{R}(\hat{X})\right), \\
& p_{R}^{* \omega}(\hat{y} ; \hat{x})=P_{\omega^{*}}^{y, s}\left(Y_{s-t}=x, s-t<\tau_{R}(\hat{Y})\right),
\end{aligned}
$$

where

$$
\tau_{R}(\hat{X}):=\inf \left\{t \geq 0: X_{t} \notin B_{R}\right\}
$$

and $\tau_{R}(\hat{Y})$ is defined similarly. Note that

$$
p_{R}^{* \omega}(\hat{y} ; \hat{x})=\frac{\rho_{\omega}(\hat{x})}{\rho_{\omega}(\hat{y})} p_{R}^{\omega}(\hat{x} ; \hat{y})
$$

Proposition 31 For any $\hat{y}=(y, s) \in B_{R} \times(0, \infty)$ and any non-negative $\omega^{*}$-caloric function $v$ on $B_{R} \times(0, s]$,

$$
\begin{aligned}
v(\hat{y}) & =\sum_{x \in \partial B_{R}, z \in B_{R}, x \sim z} \int_{0}^{s} \frac{\rho_{\omega}(x, t)}{\rho_{\omega}(\hat{y})} \omega_{t}(z, x) p_{R}^{\omega}(z, t ; \hat{y}) v(x, t) \mathrm{d} t \\
& +\sum_{x \in B_{R}} \frac{\rho_{\omega}(x, 0)}{\rho_{\omega}(\hat{y})} p_{R}^{\omega}(x, 0 ; \hat{y}) v(x, 0) .
\end{aligned}
$$

Proof Write the two summations in the proposition as I and II. Clearly, II = $E_{\omega^{*}}^{\hat{y}}\left[v\left(\hat{Y}_{S}\right) 1_{\tau_{R}>s}\right]$. Since $\left(v\left(\hat{Y}_{t}\right)\right)_{t \geq 0}$ is a martingale, we have

$$
v(y, s)=E_{\omega^{*}}^{\hat{y}}\left[v\left(\hat{Y}_{\tau_{R}}\right) 1_{\tau_{R} \leq s}\right]+E_{\omega^{*}}^{\hat{y}}\left[v\left(\hat{Y}_{s}\right) 1_{\tau_{R}>s}\right] .
$$

So it remains to show $\mathrm{I}=E_{\omega^{*}}^{y, s}\left[v\left(\hat{Y}_{\tau_{R}}\right) 1_{\tau_{R} \leq s}\right]$. We claim that for $x \in \partial B_{R}$,

$$
P_{\omega *}^{\hat{y}}\left(Y_{\tau_{R}}=x, \tau_{R} \in \mathrm{d} t\right)=\sum_{z \in B_{R}, z \sim x} \frac{\rho_{\omega}(x, s-t)}{\rho_{\omega}(\hat{y})} \omega_{s-t}(z, x) p_{R}^{\omega}(z, s-t ; \hat{y}) \mathrm{d} t .(35)
$$

Indeed, for $h>0$ small enough, $x \in \partial B_{R}$ and almost every $t \in(0, s)$,

$$
\begin{aligned}
& P_{\omega^{*}}^{y, s}\left(Y_{\tau_{R}}=x, \tau_{R} \in(t-h, t+h)\right) \\
& =\sum_{z \in B_{R}: z \sim x} P_{\omega^{*}}^{\hat{y}}\left(Y_{t-h}=z, \tau_{R}>t-h\right) P_{\omega^{*}}^{z, s-t+h}\left(Y_{2 h}=x\right)+o(h) \\
& =\sum_{z \in B_{R}: z \sim x} p_{R}^{\omega^{*}}(\hat{y} ; z, s-t) \int_{-h}^{h} \omega_{s-t+r}^{*}(z, x) \mathrm{d} r+o(h) .
\end{aligned}
$$


Dividing both sides by $2 h$ and taking $h \rightarrow 0$, display (35) follows by Lebesgue's differentiation theorem. Applying (35) to

$$
E_{\omega^{*}}^{y, s}\left[v\left(\hat{Y}_{\tau_{R}}\right) 1_{\tau_{R} \leq s}\right]=\sum_{x \in \partial B_{R}} \int_{0}^{s} v(x, s-t) P_{\omega *}^{\hat{y}}\left(Y_{\tau_{R}}=x, \tau_{R} \in \mathrm{d} t\right),
$$

we obtain $\mathrm{I}=E_{\omega^{*}}^{y, s}\left[v\left(\hat{Y}_{\tau_{R}}\right) 1_{\tau_{R} \leq s}\right]$ with a change of variable.

For fixed $\hat{y}:=(y, s) \in B_{R} \times \mathbb{R}$, set $u(\hat{x}):=p_{2 R}^{\omega}(\hat{x}, \hat{y})$. Then $\mathcal{L}_{\omega} u=0$ in $B_{2 R} \times(-\infty, s) \cup\left(B_{2 R} \backslash B_{R}\right) \times \mathbb{R}$ and $u(x, t)=0$ when $x \in \partial B_{2 R}$ or $t>s$. By Proposition 30 and Proposition 28, for any $(x, t) \in B_{2 R} \times\left(s-4 R^{2}, s-\frac{R^{2}}{2}\right)$,

$$
u(x, t) \asymp u\left(o, s-R^{2}\right) \operatorname{dist}\left(x, \partial B_{2 R}\right) / R,
$$

and, for any $(x, t) \in\left(B_{2 R} \backslash B_{3 R / 2}\right) \times\left(s-4 R^{2}, s\right)$,

$$
u(x, t) \leq C u\left(o, s-R^{2}\right) \operatorname{dist}\left(x, \partial B_{2 R}\right) / R .
$$

Lemma 32 Let $v \geq 0$ satisfies $\mathcal{L}_{\omega}^{*} v=0$ in $B_{2 R} \times\left(0,4 R^{2}\right]$, then for any $\bar{Y}=(\bar{y}, \bar{s}) \in$ $B_{R} \times\left(3 R^{2}, 4 R^{2}\right]$ and $\underline{Y}=(\underline{y}, \underline{s}) \in B_{R} \times\left(R^{2}, 2 R^{2}\right)$, we have

$$
\frac{v(\bar{Y})}{v(\underline{Y})} \geq C \frac{\int_{0}^{R^{2}} \rho_{\omega}\left(\partial B_{2 R}, t\right) \mathrm{d} t+\sum_{x \in B_{2 R}} \rho_{\omega}(x, 0) \operatorname{dist}\left(x, \partial B_{2 R}\right)}{\int_{0}^{4 R^{2}} \rho_{\omega}\left(\partial B_{2 R}, t\right) \mathrm{d} t+\sum_{x \in B_{2 R}} \rho_{\omega}(x, 0) \operatorname{dist}\left(x, \partial B_{2 R}\right)} .
$$

Proof Write $\hat{x}:=(x, t)$ and set $\bar{u}(\hat{x}):=p_{2 R}^{\omega}(\hat{x} ; \bar{Y}), \underline{u}(\hat{x}):=p_{2 R}^{\omega}(\hat{x} ; \underline{Y})$. By Proposition 31 and (36),

$$
\begin{aligned}
v(\bar{Y}) \geq & C \sum_{x \in \partial B_{2 R}, z \in B_{2 R}, x \sim z} \int_{0}^{\underline{s}} \frac{\rho_{\omega}(\hat{x})}{\rho_{\omega}(\bar{Y})} \bar{u}(z, t) v(\hat{x}) \mathrm{d} t \\
& +C \sum_{x \in B_{2 R}} \frac{\rho_{\omega}(x, 0)}{\rho_{\omega}(\bar{Y})} \bar{u}\left(0, \bar{s}-R^{2}\right) \frac{\operatorname{dist}\left(x, \partial B_{2 R}\right)}{R} v(x, 0) \\
\geq & C \frac{\bar{u}\left(0, \bar{s}-R^{2}\right)}{R \rho_{\omega}(\bar{Y})}\left[\sum_{x \in \partial B_{2 R}} \int_{0}^{\underline{s}} \rho_{\omega}(\hat{x}) v(\hat{x}) \mathrm{d} t\right. \\
& \left.+\sum_{x \in B_{2 R}} \rho_{\omega}(x, 0) \operatorname{dist}\left(x, \partial B_{2 R}\right) v(x, 0)\right]
\end{aligned}
$$


Similarly, by Proposition 31 and (37), we have

$$
\begin{aligned}
v(\underline{Y}) \leq & C \frac{\underline{u}\left(0, \underline{s}-R^{2}\right)}{R \rho_{\omega}(\underline{Y})}\left[\sum_{x \in \partial B_{2 R}} \int_{0}^{\underline{s}} \rho_{\omega}(\hat{x}) v(\hat{x}) \mathrm{d} t\right. \\
& \left.+\sum_{x \in B_{2 R}} \rho_{\omega}(x, 0) \operatorname{dist}\left(x, \partial B_{2 R}\right) v(x, 0)\right] .
\end{aligned}
$$

Combining (38) and (39), we get

$$
\frac{v(\bar{Y})}{v(\underline{Y})} \geq C \frac{\bar{u}\left(o, \bar{s}-R^{2}\right) / \rho_{\omega}(\bar{Y})}{\underline{u}\left(o, \underline{s}-R^{2}\right) / \rho_{\omega}(\underline{Y})} .
$$

Next, applying Proposition 31 to the constant function 1 and using (37),

$$
\begin{aligned}
1 & =\sum_{x \in \partial B_{2 R}, z \in B_{2 R}, z \sim x} \int_{0}^{\bar{s}} \frac{\rho_{\omega}(\hat{x})}{\rho_{\omega}(\bar{Y})} \omega_{t}(z, x) \bar{u}(z, t) \mathrm{d} t+\sum_{x \in B_{2 R}} \frac{\rho_{\omega}(x, 0)}{\rho_{\omega}(\bar{Y})} \bar{u}(x, 0) \\
& \leq C \frac{\bar{u}\left(o, \bar{s}-R^{2}\right)}{R \rho_{\omega}(\bar{Y})}\left[\sum_{x \in \partial B_{2 R}} \int_{0}^{\bar{s}} \rho_{\omega}(\hat{x}) \mathrm{d} t+\sum_{x \in B_{2 R}} \rho_{\omega}(x, 0) \operatorname{dist}\left(x, \partial B_{2 R}\right)\right] .
\end{aligned}
$$

Similarly, by Proposition 31 and (36),

$$
1 \geq C \frac{\underline{u}\left(o, \underline{s}-R^{2}\right)}{R \rho_{\omega}(\underline{Y})}\left[\sum_{x \in \partial B_{2 R}} \int_{0}^{\underline{s} / 2} \rho_{\omega}(\hat{x}) \mathrm{d} t+\sum_{x \in B_{2 R}} \rho_{\omega}(x, 0) \operatorname{dist}\left(x, \partial B_{R}\right)\right] .
$$

These inequalities, together with (40), yield the lemma.

Remark 33 It is clear that for static environments, the adjoint Harnack inequality (Theorem 6) follows immediately from Lemma 32. However, in time-dependent case, we need the parabolic volume-doubling property of $\rho_{\omega}$.

Proof of Theorem 6 First, we will show that for all $R>0$,

$$
\begin{aligned}
& \int_{0}^{s} \rho_{\omega}\left(\partial B_{R}, t\right) \mathrm{d} t+\sum_{x \in B_{R}} \rho_{\omega}(x, 0) \operatorname{dist}\left(x, \partial B_{R}\right) \\
& \asymp \frac{1}{R} \int_{0}^{s} \rho_{\omega}\left(B_{R}, t\right) \mathrm{d} t+\sum_{x \in B_{R}} \rho_{\omega}(x, s) \operatorname{dist}\left(x, \partial B_{R}\right) .
\end{aligned}
$$


Recall $\tau_{R}$ at (34) and set $g(x, t)=E_{\omega}^{x, t}\left[\tau_{R}(\hat{X})\right]$. Note that $g(x, \cdot)=0$ for $x \notin B_{R}$ and $\mathcal{L}_{\omega} g(x, t)=-1$ if $x \in B_{R}$. By (3), for any $s>0$,

$$
\begin{aligned}
0= & \sum_{x \in \mathbb{Z}^{d}} \int_{0}^{s} g(x, t)\left[\sum_{y} \rho_{\omega}(y, t) \omega_{t}(y, x)-\partial_{t} \rho_{\omega}(x, t)\right] \mathrm{d} t \\
= & \sum_{x \in \partial B_{R}, y \in B_{R}} \int_{0}^{s} \rho(x, t) \omega_{t}(x, y) g(y, t) \mathrm{d} t+\sum_{x \in B_{R}} g(x, 0) \rho(x, 0) \\
& -\sum_{x \in B_{R}} \int_{0}^{s} \rho(x, t) \mathrm{d} t-\sum_{x \in B_{R}} g(x, s) \rho(x, s) .
\end{aligned}
$$

Moreover, since $\left|X_{t}\right|^{2}-\frac{d}{\kappa} t$ and $\left|X_{t}\right|^{2}-\kappa t$ are super- and sub- martingales,

$$
g(x, t) \asymp E_{\omega}^{x, t}\left[\left|X_{\tau_{R}}\right|^{2}-|x|^{2}\right] \asymp R \operatorname{dist}\left(x, \partial B_{R}\right) \quad \forall(x, t) \in B_{R} \times \mathbb{R}
$$

by the optional-stopping theorem. Display (41) then follows.

Combining (41) and Lemma 32, we obtain

$$
\frac{v(\bar{Y})}{v(\underline{Y})} \geq C \frac{\int_{0}^{R^{2}} \rho\left(B_{2 R}, t\right) \mathrm{d} t+R \sum_{x \in B_{2 R}} \rho\left(x, R^{2}\right) \operatorname{dist}\left(x, \partial B_{2 R}\right)}{\int_{0}^{4 R^{2}} \rho\left(B_{2 R}, t\right) \mathrm{d} t+R \sum_{x \in B_{2 R}} \rho\left(x, 4 R^{2}\right) \operatorname{dist}\left(x, \partial B_{2 R}\right)} .
$$

Finally, Theorem 6 follows by Theorem 8 and the above inequality.

\section{Proof of Theorems 4, 11, and Corollary 12}

The goal of this section is to prove the LLT (Theorem 4), the HKE (Theorem 11), and Corollary 12 . With the QCLT and the Hölder regularity for $\omega^{*}$-caloric functions, the LLT, quenched heat kernel bound (6), and the Green's function asymptotics Corollary 12(ii)(iii) all follow from rather standard arguments, which have been successfully implemented for random conductance models, e.g., [2,3,5,7]. Our main novelty in this section is the bounds (7)(8) of positive and negative moments for the heat kernel.

\subsection{Proof of Theorem 11}

Proof First, using Theorem 6 and standard arguments, we will prove (6). Recall that $v(\hat{x}):=q^{\omega}(\hat{0}, \hat{x})$ satisfies $\mathcal{L}_{\omega}^{*} v=0$ in $\mathbb{Z}^{d} \times(0, \infty)$. By Theorem 6 , for $\hat{x}=(x, t) \in$ $\mathbb{Z}^{d} \times(0, \infty)$, we have $v(\hat{x}) \leq C \min _{y \in B_{\sqrt{t}}(x)} v(y, 3 t)$ and so

$$
\begin{aligned}
v(\hat{x}) & \leq \frac{C}{\rho\left(B_{\sqrt{t}}(x), 3 t\right)} \sum_{y \in B_{\sqrt{t}}(x)} \rho(y, 3 t) v(y, 3 t) \\
& =\frac{C}{\rho\left(B_{\sqrt{t}}(x), 3 t\right)} P_{\omega}^{0,0}\left(X_{3 t} \in B_{\sqrt{t}}(x)\right) \leq C \exp [-c \mathfrak{h}(|x|, t)],
\end{aligned}
$$


where Corollary 14 is used in the last inequality. Moreover, for any $s \in[0, t],|y| \leq$ $|x|+c \sqrt{t}$, by Theorem 8 and iteration,

$$
\rho\left(B_{\sqrt{t}}(x), 3 t\right) \geq C \rho\left(B_{\sqrt{t \vee 1}}(x), s\right) \geq C\left(\frac{|x|}{\sqrt{t \vee 1}}+1\right)^{-c} \rho\left(B_{\sqrt{t \vee 1}}(y), s\right) .
$$

Since $\frac{|x|}{\sqrt{t \vee 1}}+1 \leq C_{\epsilon} e^{\epsilon \mathfrak{h}(|x|, t)}$ for any $\epsilon>0$, the upper bound in (6) follows.

To obtain the lower bound in (6), by similar argument as above and Theorem 6 , $v(\hat{x}) \geq C \max _{y \in B \sqrt{t} / 2}(x) v(y, t / 4)$ for $\hat{x} \in \mathbb{Z}^{d} \times(0, \infty)$, and so

$$
v(\hat{x}) \geq \frac{C}{\rho\left(B_{\sqrt{t} / 2}(x), t / 4\right)} P_{\omega}^{0,0}\left(X_{t / 4} \in B_{\sqrt{t} / 2}(x)\right) .
$$

We claim that for any $(y, s) \in \mathbb{Z}^{d} \times(0, \infty)$,

$$
P_{\omega}^{y, 0}\left(X_{s} \in B_{\sqrt{s}}\right) \geq C e^{-c|y|^{2} / s} .
$$

Indeed, the case $|y| / \sqrt{s} \leq 3$ follows from Lemma 15. When $|y| / \sqrt{s}>3$, let

$$
n=\left\lfloor 2|y|^{2} / s\right\rfloor \text {. }
$$

Set $u(x, t):=p^{\omega}\left(x, t ; B_{\sqrt{s}}, s\right)$. Then $u$ is $\omega$-caloric on $\mathbb{Z}^{d} \times(-\infty, s)$. Taking a sequence of points $\left(y_{i}\right)_{i=1}^{n}$ such that $y_{0}=y, y_{n}=0$ and $\left|y_{i}-y_{i+1}\right| \leq|y| / n$, for $i=0, \ldots n-1$,

$$
\begin{aligned}
& \min _{x \in B_{|y| / \sqrt{n}}\left(y_{i}\right)} u\left(x, \frac{i|y|^{2}}{n^{2}}\right) \\
& \geq \min _{z \in B_{|y| / \sqrt{n}}\left(y_{i}\right)} p^{\omega}\left(z, \frac{i|y|^{2}}{n^{2}} ; B_{\left.|y| / \sqrt{n}\left(y_{i+1}\right), \frac{(i+1)|y|^{2}}{n^{2}}\right)} \min _{x \in B_{|y| / \sqrt{n}}\left(y_{i+1}\right)} u\left(x, \frac{(i+1)|y|^{2}}{n^{2}}\right)\right. \\
& \stackrel{\text { Lemma } 15}{ }{ }^{\geq} \min _{x \in B_{|y| / \sqrt{n}}\left(y_{i+1}\right)} u\left(x, \frac{(i+1)|y|^{2}}{n^{2}}\right) .
\end{aligned}
$$

Iteration then yields $u(y, 0) \geq C^{n-1} \min _{x \in B_{|y| / \sqrt{n}}} u\left(x, \frac{|y|^{2}}{n}\right) \stackrel{\text { Lemma } 15}{\geq} C^{n}$. Inequality (43) is proved. Then, by (42),

$$
v(x, t) \geq \frac{C}{\rho\left(B_{\sqrt{t} / 2}(x), t / 4\right)} e^{-c|x|^{2} / t} .
$$

Moreover, by Theorem 8 , we have for any $s \in[0, t],|y| \leq|x|$,

$$
\rho\left(B_{\sqrt{t} / 2}(x), t / 4\right) \leq C \rho\left(B_{\sqrt{t} / 2}(x), s\right) \leq C\left(\frac{|x|}{\sqrt{t}}+1\right)^{c} \rho\left(B_{\sqrt{t}}(y), s\right) .
$$

The lower bound in (6) is proved. 
Next, we will prove the moment bounds (7) and (8), which, by (6) and (22), are equivalent to showing that, for $r:=\sqrt{t}>0$,

$$
\left\|\frac{\rho(\hat{0})}{\rho\left(Q_{r}\right)}\right\|_{L^{(d+1) / d}(\mathbb{P})} \leq C r^{2}(r \vee 1)^{-d} \text { and }\left\|\frac{\rho(\hat{0})}{\rho\left(Q_{r}\right)}\right\|_{L^{-p}(\mathbb{P})} \geq C r^{2}(r \vee 1)^{-d},
$$

where $Q_{r}$ is as defined in (18). Indeed, using the translation-invariance of $\mathbb{P}$ and the volume-doubling property of $\rho$, for $q:=(d+1) / d$,

$$
\left\|\rho(\hat{0}) / \rho\left(Q_{r}\right)\right\|_{L^{q}(\mathbb{P})}^{q} \leq C \frac{1}{\left|Q_{r}\right|} \int_{\hat{x} \in Q_{r}} E_{\mathbb{P}}\left[\frac{\rho(\hat{x})^{q}}{\rho\left(Q_{r}\right)^{q}}\right] \leq C /\left|Q_{r}\right|^{q},
$$

where we used the Reverse Hölder inequality (Corollary 24(i)) in the last inequality. Recalling (24), inequality (7) then follows from the fact that $\left|Q_{r}\right|=r^{2} \sum_{x \in B_{r}} 1 \asymp$ $r^{2}(r \vee 1)^{-d}$.

To obtain (8), note that by translation invariance and $\mathbb{P}$ and the volume-doubling property of $\rho$, taking $\epsilon \in(0,1 /(p-1))$,

$$
\left\|\rho\left(Q_{r}\right) / \rho(\hat{0})\right\|_{L^{\epsilon}(\mathbb{P})}^{\epsilon} \leq \frac{C}{\left|Q_{r}\right|} E_{\mathbb{P}}\left[\int_{\hat{x} \in Q_{r}} \frac{\rho\left(Q_{r}\right)^{\epsilon}}{\rho(\hat{x})^{\epsilon}}\right] \leq C\left|Q_{r}\right|^{\epsilon},
$$

where we used the $A_{p}$ inequality (Theorem 26(a)) of $\rho$ in the last inequality. Therefore $\left\|\rho(\hat{0}) / \rho\left(Q_{r}\right)\right\|_{L^{-\epsilon}(\mathbb{P})} \geq C r^{2}(r \vee 1)^{-d}$ and (8) is proved.

Display (9) follows from (7), (8), and Minkowski's integral inequality.

\subsection{Proof of Theorem 4 and Corollary 12}

Recall $q^{\omega}(\hat{y}, \hat{x})$ in (4). For any $\hat{x}=(x, t) \in \mathbb{R}^{d} \times \mathbb{R}$, set

$$
v(\hat{x}):=q^{\omega}(\hat{0} ;\lfloor x\rfloor, t),
$$

where $\lfloor x\rfloor$ is as in Theorem 4 . Note that $\mathcal{L}_{\omega}^{*} v=0$ in $\mathbb{Z}^{d} \times(0, \infty)$. By Corollary 7 and Theorem 11 , for any $\hat{y}=(y, s) \in B_{\sqrt{t}}(x) \times\left(\frac{t}{2}, t\right)$,

$$
\begin{aligned}
|v(\hat{x})-v(\hat{y})| & \leq C\left(\frac{|x-y|+\sqrt{t-s}}{\sqrt{t}}\right)^{\gamma} \sup _{B_{\sqrt{t}}(x) \times\left(\frac{t}{2}, t\right]} v \\
& \leq C\left(\frac{|x-y|+\sqrt{t-s}}{\sqrt{t}}\right)^{\gamma} t^{-d / 2}
\end{aligned}
$$

when $t>t_{0}(\omega)$ is big enough. Here in the last inequality we used Corollary 12(i) which is an immediate consequence of Theorem 11 and (23).

Recall $\mathcal{O}_{r}$ in (27). For $\hat{x}=(x, t) \in \mathbb{R}^{d} \times \mathbb{R}$, write

$$
\hat{x}^{n}:=\left(\lfloor n x\rfloor, n^{2} t\right) .
$$


To prove Theorem 4, it suffices to show that for any $K>T$,

$$
\lim _{n \rightarrow \infty} \sup _{\hat{x} \in \mathcal{O}_{K} \times[T, K]}\left|n^{d} v\left(\hat{x}^{n}\right)-p_{t}^{\Sigma}(0, x)\right|=0 .
$$

Indeed, for any $\epsilon>0$, there exists $K=K(T, \epsilon, d, \kappa)>0$ such that, writing $\mathscr{D}:=$ $\left(\mathbb{R}^{d} \times[T, \infty)\right) \backslash\left(\mathcal{O}_{K} \times[T, K]\right)$, we have

$$
\varlimsup_{n \rightarrow \infty} \sup _{\mathscr{D}} n^{d} v\left(\hat{x}^{n}\right)+p_{t}^{\Sigma}(0, x) \stackrel{(6),(23)}{\leq} C \sup _{\mathscr{D}} t^{-d / 2} e^{-c|x|^{2} / t} \leq \epsilon .
$$

Hence Theorem 4 follows provided that (45) is proved.

Proof of Theorem 4 As we discussed in the above, it suffices to prove (45). For any $\epsilon>0$,

$$
\left|n^{d} v\left(\hat{x}^{n}\right)-p_{t}^{\Sigma}(0, x)\right| \leq A(\hat{x}, \epsilon)+B_{n}(\hat{x}, \epsilon)+C_{n}(\hat{x}, \epsilon)
$$

where $A(\hat{x}, \epsilon)=\left|\frac{\int_{t}^{t+\epsilon^{2}} p_{s}^{\Sigma}\left(0, \mathcal{O}_{\epsilon}(x)\right) \mathrm{d} s}{\epsilon^{2}\left|\mathcal{O}_{\epsilon}\right|}-p_{t}^{\Sigma}(0, x)\right|$,

$$
\begin{aligned}
& B_{n}(\hat{x}, \epsilon)=\left|\int_{t}^{t+\epsilon^{2}} \frac{P_{\omega}^{\hat{0}}\left(X_{n^{2} s} \in n \mathcal{O}_{\epsilon}(x)\right)-p_{s}^{\Sigma}\left(0, \mathcal{O}_{\epsilon}(x)\right)}{\epsilon^{2}\left|\mathcal{O}_{\epsilon}\right|} \mathrm{d} s\right|, \\
& C_{n}(\hat{x}, \epsilon)=\left|n^{d} v\left(\hat{x}^{n}\right)-\frac{\int_{t}^{t+\epsilon^{2}} P_{\omega}^{\hat{0}}\left(X_{n^{2} s} \in n \mathcal{O}_{\epsilon}(x)\right) \mathrm{d} s}{\epsilon^{2}\left|\mathcal{O}_{\epsilon}\right|}\right| .
\end{aligned}
$$

First, we will show that

$$
\varlimsup_{n \rightarrow \infty} \sup _{\hat{x} \in \mathcal{O}_{K} \times[T, K]} C_{n}(\hat{x}, \epsilon)=O\left(\epsilon^{\gamma}\right) .
$$

To this end, note that there exists $N=N(T, \omega, d, \kappa)$ such that for $n \geq N$,

$$
\begin{aligned}
C_{n}(\hat{x}, \epsilon) \leq & n^{d} v\left(\hat{x}^{n}\right)\left|1-\frac{\int_{t}^{t+\epsilon^{2}} \rho\left(n \mathcal{O}_{\epsilon}(x), n^{2} s\right) \mathrm{d} s}{\epsilon^{2}\left|n \mathcal{O}_{\epsilon}\right|}\right| \\
+ & \sum_{y \in n \mathcal{O}_{\epsilon}(x)} \int_{t}^{t+\epsilon^{2}}\left|v\left(y, n^{2} s\right)-v\left(\hat{x}^{n}\right)\right| \rho\left(y, n^{2} s\right) \mathrm{d} s /\left(\epsilon^{2}\left|\mathcal{O}_{\epsilon}\right|\right) \\
\leq & C T^{-d / 2}\left|1-\frac{\int_{t}^{t+\epsilon^{2}} \rho\left(n \mathcal{O}_{\epsilon}(x), n^{2} s\right) \mathrm{d} s}{\epsilon^{2}\left|n \mathcal{O}_{\epsilon}\right|}\right| \\
& +C T^{-(\gamma+d) / 2} \epsilon^{\gamma} \int_{t}^{t+\epsilon^{2}} \rho\left(n \mathcal{O}_{\epsilon}(x), n^{2} s\right) \mathrm{d} s /\left(\epsilon^{2}\left|n \mathcal{O}_{\epsilon}\right|\right),
\end{aligned}
$$


where in the second inequality we used Corollary 12(i) and (44). Further, by an ergodic theorem of Krengel and Pyke [21, Theorem 1] and (2),

$$
\lim _{n \rightarrow 0} \sup _{\hat{x} \in \mathcal{O}_{K} \times[T, K]}\left|1-\frac{\int_{t}^{t+\epsilon^{2}} \rho\left(n \mathcal{O}_{\epsilon}(x), n^{2} s\right) \mathrm{d} s}{\epsilon^{2}\left|n \mathcal{O}_{\epsilon}\right|}\right|=0 .
$$

Display (47) follows.

Next, for $\hat{x}=(x, t)$, by writing $B_{n}(\hat{x}, \epsilon)$ as

$$
\left|\frac{\int_{t}^{t+\epsilon^{2}} P_{\omega}^{\hat{0}}\left(X_{n^{2} s} \in n \mathcal{O}_{\epsilon}(x)\right) \mathrm{d} s}{\epsilon^{2}\left|\mathcal{O}_{\epsilon}\right|}-\frac{\int_{t}^{t+\epsilon^{2}} p_{s}^{\Sigma}\left(0, \mathcal{O}_{\epsilon}(x)\right) \mathrm{d} s}{\epsilon^{2}\left|\mathcal{O}_{\epsilon}\right|}\right|=:\left|B_{n}^{1}(\hat{x}, \epsilon)-B^{2}(\hat{x}, \epsilon)\right|,
$$

we will show that

$$
\varlimsup_{n \rightarrow \infty} \sup _{\hat{x} \in \mathcal{O}_{K} \times[T, K]} B_{n}(\hat{x}, \epsilon)=O\left(\epsilon^{\gamma}\right) .
$$

We claim that $B_{n}(\hat{x}, \epsilon)$ is approximately equicontinuous (with order $\epsilon^{\gamma}$ ). That is, there exist $N, \delta$ depending on $(\epsilon, \omega, d, \kappa, T, K)$ such that, whenever $n \geq N$ and $\hat{x}_{1}=$ $\left(x_{1}, t_{1}\right), \hat{x}_{2}=\left(x_{2}, t_{2}\right) \in \mathcal{O}_{K} \times[T, K]$ satisfy $\left|\hat{x}_{1}-\hat{x}_{2}\right|_{1}:=\left|x_{1}-x_{2}\right|+\left|t_{1}-t_{2}\right|<\delta$, we have

$$
\left|B_{n}\left(\hat{x}_{1}, \epsilon\right)-B_{n}\left(\hat{x}_{2}, \epsilon\right)\right|<C \epsilon^{\gamma} .
$$

It suffices to show that $B_{n}^{1}(\hat{x}, \epsilon)$ is approximately equicontinuous. Indeed, by (47) and (44), when $n \geq N$ is large and $\hat{x}_{1}, \hat{x}_{2} \in \mathcal{O}_{K} \times[T, K]$,

$$
\begin{aligned}
\left|B_{n}^{1}\left(\hat{x}_{1}, \epsilon\right)-B_{n}^{1}\left(\hat{x}_{2}, \epsilon\right)\right| & \leq C_{n}\left(\hat{x}_{1}, \epsilon\right)+C_{n}\left(\hat{x}_{2}, \epsilon\right)+n^{d}\left|v\left(\hat{x}_{1}^{n}\right)-v\left(\hat{x}_{2}^{n}\right)\right| \\
& \leq C \epsilon^{\gamma}+C\left(\left|x_{1}-x_{2}\right|+\sqrt{\left|t_{1}-t_{2}\right|}\right)^{\gamma} .
\end{aligned}
$$

The approximate equicontinuity of $B_{n}^{1}(\hat{x}, \epsilon)$ follows. To prove (49), we choose a finite sequence $\left\{\hat{x}_{i}\right\}_{i=1}^{M}$ such that $\min _{1 \leq i \leq M}\left|\hat{x}-\hat{x}_{i}\right|_{1}<\delta$ for all $\hat{x} \in \mathcal{O}_{K} \times[T, K]$. Since $\lim _{n \rightarrow \infty} \max _{1 \leq i \leq M} B_{n}\left(\hat{x}_{i}\right)=0$ by the QCLT (Theorem 1), display (49) follows by the approximate equicontinuity.

Clearly, $\lim _{\epsilon \rightarrow 0} \sup _{\hat{x} \in \mathcal{O}_{K} \times[T, K]} A(\hat{x}, \epsilon)=0$. This, together with (47) and (49), yields the uniform convergence of (46) by sending first $n \rightarrow \infty$ and then $\epsilon \rightarrow 0$. Our proof of Theorem 4 is complete.

Proof of Corollary 12: (i) follows from Theorem 11 and (23). (ii) and (iii) are consequences of Theorems 4 and 11. Their proofs, which are similar to [2, Theorem 1.14] and [5, Theorem 1.4], can be found in Sect. A.6.

Acknowledgements We thank two anonymous referees for their careful reading of our paper and their valuable comments. XG did the main part of his work while at the University of Wisconsin-Madison. He thanks Professors Timo Sepäläinen, Hung Vinh Tran, and other colleagues at the UW for their hospitality and supportive environment they created. 
Funding Open Access funding enabled and organized by Projekt DEAL.

Open Access This article is licensed under a Creative Commons Attribution 4.0 International License, which permits use, sharing, adaptation, distribution and reproduction in any medium or format, as long as you give appropriate credit to the original author(s) and the source, provide a link to the Creative Commons licence, and indicate if changes were made. The images or other third party material in this article are included in the article's Creative Commons licence, unless indicated otherwise in a credit line to the material. If material is not included in the article's Creative Commons licence and your intended use is not permitted by statutory regulation or exceeds the permitted use, you will need to obtain permission directly from the copyright holder. To view a copy of this licence, visit http://creativecommons.org/licenses/by/4.0/.

\section{A Appendix}

In Sect. A.1 we will show properties (i)-(iii) of $\rho_{\omega}$ in Remark 3. In Sect. A.2, we prove the Hölder estimate (Corollary 7) for $\omega^{*}$-caloric functions. Sections A.3 and A.4 are devoted to the maximum principle (which is used in various places in the paper) and the mean value inequality (used in the proof of the PHI Proposition 5) for $\mathcal{L}_{\omega}$, respectively. Section A.5 contains the proof of Lemma 25. Corollary 12(ii)(iii) are proved in Sect. A.6.

\section{A.1 Properties (i)-(iii) in Remark 3}

Proof (i)Since $\mathbb{Q}$ is an invariant measure for $\left(\bar{\omega}_{t}\right)$, we have for any bounded measurable function $f$ on $\Omega, y \in \mathbb{Z}^{d}, \hat{x}=(x, t)$, and $s<t$,

$$
\begin{aligned}
0 & =E_{\mathbb{Q}} E_{\omega}^{0,0}\left[f\left(\theta_{-\hat{x}}\left(\bar{\omega}_{t-s}\right)\right)-f\left(\theta_{-\hat{x}} \omega\right)\right] \\
& =E_{\mathbb{P}}\left[\rho(\omega) \sum_{y \in \mathbb{Z}^{d}} p^{\omega}(0,0 ; x-y, t-s)\left[f\left(\theta_{-y,-s} \omega\right)-f\left(\theta_{-\hat{x}} \omega\right)\right]\right] \\
& =E_{\mathbb{P}}\left[f(\omega)\left[\sum_{y \in \mathbb{Z}^{d}} \rho\left(\theta_{\hat{y}} \omega\right) p^{\omega}(\hat{y}, \hat{x})-\rho\left(\theta_{\hat{x}} \omega\right)\right]\right]
\end{aligned}
$$

where $\hat{y}=(y, s)$ and we used the translation-invariance of $\mathbb{P}$ in the last equality. Moreover, by Fubini's theorem, for any bounded compactly-supported continuous function $\phi: \mathbb{R} \rightarrow \mathbb{R}$,

$$
E_{\mathbb{P}}\left[f(\omega) \int_{-\infty}^{t} \phi(s)\left[\sum_{y \in \mathbb{Z}^{d}} \rho\left(\theta_{\hat{y}} \omega\right) p^{\omega}(\hat{y}, \hat{x})-\rho\left(\theta_{\hat{x}} \omega\right)\right] \mathrm{d} s\right]=0
$$

Thus we have that $\mathbb{P}$-almost surely, for any such test function $\phi$ on $\mathbb{R}$,

$$
\int_{-\infty}^{t} \phi(s)\left[\sum_{y \in \mathbb{Z}^{d}} \rho_{\omega}(\hat{y}) p^{\omega}(\hat{y}, \hat{x})-\rho_{\omega}(\hat{x})\right] \mathrm{d} s=0,
$$


which (together with the translation-invariance of $\mathbb{P}$ ) implies that $\mathbb{P}$-almost surely, $\rho_{\omega}(x, t) \delta_{x} \mathrm{~d} t$ is an invariant measure for the process $\left(\hat{X}_{t}\right)_{t \geq 0}$.

(ii) We have $\rho_{\omega}>0$ since the measures $\mathbb{Q}$ and $\mathbb{P}$ are equivalent. The uniqueness follows from the uniqueness of $\mathbb{Q}$ in [14, Theorem 2.1(iii)].

(iii) By (50) and Fubini's theorem, we also have that $\mathbb{P}$-almost surely, for any test function $\phi(t)$ as in (i) and any $h>0, x \in \mathbb{Z}^{d}$,

$\int_{-\infty}^{\infty} \phi(t)\left[\sum_{y \in \mathbb{Z}^{d}} \rho_{\omega}(y, t)\left(p^{\omega}(y, t ; x, t+h)-\delta_{x}(y)\right)-\left(\rho_{\omega}(x, t+h)-\rho_{\omega}(\hat{x})\right] \mathrm{d} t=0\right.$.

Dividing both sides by $h$ and letting $h \rightarrow 0$, we obtain (3) with $\partial_{t} \rho_{\omega}$ replaced by the weak derivative. Note that the weak differentiability of $\rho_{\omega}$ in $t$ implies that it has an absolutely continuous (in $t$ ) version. Since $\rho_{\omega}$ is only used as a density, we may always assume that $\mathbb{P}$-almost surely, $\rho_{\omega}(x, \cdot)$ is continuous and almost-everywhere differentiable in $t$.

\section{A.2 Proof of Corollary 7}

Proof Assume $\left(x_{0}, t_{0}\right)=(0,0)$ and fix $R>0$. Let $R_{k}=2^{-k} R$ and $Q^{k}=B_{R_{k}} \times$ $\left(-R_{k}^{2}, 0\right]$. Note that $Q^{k+1} \subset Q^{k}$. For any bounded subset $E \subset \mathbb{Z}^{d} \times \mathbb{R}$, denote $\operatorname{osc}_{E} u:=\sup _{E} u-\inf _{E} u$. Set

$$
v_{k}:=\left(u-\inf _{Q^{k}} u\right) / \operatorname{osc}_{Q^{k}} u .
$$

Notice that $\inf _{Q^{k}} v_{k}=0, \sup _{Q^{k}} v_{k}=1$ and

$$
\operatorname{osc}_{Q^{k+1}} u=\operatorname{osc}_{Q^{k+1}} v_{k} \cdot \operatorname{osc}_{Q^{k}} u .
$$

We claim that $\operatorname{osc}_{Q^{k+1}} v_{k} \leq 1-\delta$ for some $\delta=\delta(d, \kappa) \in(0,1)$. Indeed, replacing $v_{k}$ by $1-v_{k}$ if necessary, we can assume $\sup _{B_{R_{k+1}} \times\left(-\frac{3}{4} R_{k}^{2},-\frac{1}{2} R_{k}^{2}\right)} v_{k} \geq 1 / 2$. By the PHI for $\mathcal{L}_{\omega}^{*}$ (Theorem 6),

$$
\inf _{Q^{k+1}} v_{k} \geq c \sup _{B_{R_{k+1}} \times\left(-\frac{3}{4} R_{k}^{2}, \frac{1}{2} R_{k}^{2}\right)} v_{k} \geq \frac{c}{2}:=\delta \in(0,1)
$$

and so osc $Q^{k+1} v_{k} \leq \sup _{Q^{k}} v_{k}-\inf _{Q^{k+1}} v_{k} \leq 1-\delta$. The claim is proved. So

$$
\operatorname{osc}_{Q^{k+1}} u \leq(1-\delta) \operatorname{osc}_{Q^{k}} u .
$$

If $r>R / 2$, the Corollary is trivial. If $r \leq R / 2$, we iterate the above inequality $k=\left\lfloor\log _{2}(R / r)\right\rfloor$ times (so that $\left.Q^{k+1} \subset B_{r} \times\left(-r^{2}, 0\right] \subset Q^{k}\right)$ to obtain

$$
\operatorname{osc}_{B_{r} \times\left(-r^{2}, 0\right]} u \leq \operatorname{osc}_{Q^{k}} u \leq(1-\delta)^{k} \operatorname{osc}_{Q^{0}} u \leq(1-\delta)^{-1}(r / R)^{\gamma} \operatorname{osc}_{Q^{0}} u .
$$

where $\gamma=-\log _{2}(1-\delta)$. Our proof is complete. 


\section{A.3 Parabolic maximum principle}

In what follows we will prove a maximum principle for parabolic difference operators under the discrete space and continuous time setting. Its statement is a tiny modification from [14, Theorem 5.1] where its proof, which follows verbatim the lines of [14, Theorem 2.2], was omitted. For the purpose of completeness we will include a full proof in the below.

For any $\mathscr{D} \subset B_{R} \times(0, T), \hat{x}:=(x, t) \in \mathscr{D}$ and $u: \mathscr{D} \cup \partial^{\mathrm{P}} \mathscr{D} \rightarrow \mathbb{R}$, define

$$
\begin{gathered}
I_{u}(\hat{x}):=\left\{p \in \mathbb{R}^{d}: u(\hat{x})-u(y, s) \geq p \cdot(x-y), \forall(y, s) \in \mathscr{D} \cup \partial^{\mathrm{P}} \mathscr{D} \text { with } s>t\right\}, \\
\Gamma=\Gamma(u, \mathscr{D}):=\left\{(x, t) \in \mathscr{D}: I_{u}(x, t) \neq \emptyset\right\}, \\
\Gamma^{+}=\Gamma^{+}(u, \mathscr{D})=\left\{\hat{x} \in \Gamma: R|p|<u(\hat{x})-p \cdot x \text { for some } p \in I_{u}(\hat{x})\right\} .
\end{gathered}
$$

Theorem A.3.1 (Maximum principle) Let $\omega \in \Omega_{\kappa}$. Recall $\int_{\mathscr{D}}$ in (24). Assume that $\mathscr{D} \subset B_{R} \times(0, T)$ is an open subset of $\mathbb{Z}^{d} \times \mathbb{R}$ for some $R, T>0$. Let $f$ be a measurable function on $\mathscr{D}$. For any function $u: \mathscr{D} \cup \partial^{\mathrm{P}} \mathscr{D} \rightarrow \mathbb{R}$ that solves $\mathcal{L}_{a} u \geq-f$ in $\mathscr{D}$, we have

$$
\sup _{\mathscr{D}} u \leq \sup _{\partial^{\mathrm{P}} \mathscr{D}} u+C R^{d /(d+1)}\left(\int_{\Gamma^{+}}|f|^{d+1}\right)^{1 /(d+1)}
$$

Proof Without loss of generality, assume $f \geq 0, \sup _{\partial^{\mathrm{P}} \mathscr{D}} u=0$, and

$$
\sup _{\mathscr{D}} u:=M>0 \text {. }
$$

Let $\Lambda=\left\{(\xi, h) \in \mathbb{R}^{d+1}: R|\xi|<h<M / 2\right\}$. For $(x, t) \in \mathscr{D}$, define a set

$$
\chi(x, t)=\left\{(p, u(x, t)-x \cdot p): p \in I_{u}(x, t)\right\} \subset \mathbb{R}^{d+1} .
$$

First, we claim that

$$
\Lambda \subset \chi\left(\Gamma^{+}\right):=\bigcup_{(x, t) \in \Gamma^{+}} \chi(x, t) .
$$

This will be proved by showing that for any $(\xi, h) \in \Lambda$, we have $(\xi, h) \in \chi\left(x_{1}, t_{1}\right)$ for some $\left(x_{1}, t_{1}\right) \in \Gamma^{+}$. Indeed, fix $(\xi, h) \in \Lambda$ and define

$$
\phi(x, t):=u(x, t)-\xi \cdot x-h .
$$

Since $\sup _{\mathscr{D}} \phi \geq M-|\xi| R-h>0$, there exists $\left(x_{0}, t_{0}\right) \in \mathscr{D}$ with $\phi\left(x_{0}, t_{0}\right)>0$. Now for any $x \in \mathbb{Z}^{d}$, set (with the convention $\sup \emptyset=-\infty$ )

$$
N_{x}=\sup \{t:(x, t) \in \mathscr{D} \text { and } \phi(x, t) \geq 0\},
$$


and let $\left(x_{1}, t_{1}\right)$ be such that

$$
t_{1}=N_{x_{1}}=\max _{x \in B_{R}} N_{x} \geq N_{x_{0}} \geq t_{0}
$$

By the continuity of $\phi$, we get $\phi\left(x_{1}, t_{1}\right) \geq 0$ and $\left(x_{1}, t_{1}\right) \in \mathscr{D} \cup \partial^{\mathrm{P}} \mathscr{D}$. Since $\left.\phi\right|_{\partial^{\mathrm{P}} \mathscr{D}}<$ 0 , we have $\left(x_{1}, t_{1}\right) \in \mathscr{D}$. Moreover, since $\mathscr{D}$ is an open set, we can conclude that $\phi\left(x_{1}, t_{1}\right)=0$ and $\phi\left(x_{1}, s\right)<0$ for all $s>t_{1}$ with $\left(x_{1}, s\right) \in \mathscr{D} \cup \partial^{\mathrm{P}} \mathscr{D}$. Hence $\xi \in I_{u}\left(x_{1}, t_{1}\right)$ and $u\left(x_{1}, t_{1}\right)-\xi \cdot x_{1}=h>R|\xi|$, which implies that $(\xi, h) \in \chi\left(x_{1}, t_{1}\right)$ and $\left(x_{1}, t_{1}\right) \in \Gamma^{+}$. Display (52) is proved.

Next, setting

$$
\chi\left(\Gamma^{+}, x\right):=\bigcup_{s:(x, s) \in \Gamma^{+}} \chi(x, s)
$$

we will show that

$$
\operatorname{Vol}_{d+1}\left(\chi\left(\Gamma^{+}, x\right)\right) \leq C \int_{0}^{T}(f(x, t) / \varepsilon)^{d+1} 1_{(x, t) \in \Gamma^{+}} \mathrm{d} t
$$

where $\operatorname{Vol}_{d+1}$ is the volume in $\mathbb{R}^{d+1}$. To this end, let $\tilde{\chi}(x, t)=I_{u}(x, t) \times\{u(x, t)\} \subset$ $\mathbb{R}^{d+1}$. Noting that, for fixed $x$, the map $(y, s) \mapsto(y, s+y \cdot x)$ is volume preserving, we then have

$$
\begin{aligned}
\operatorname{Vol}_{d+1}\left(\chi\left(\Gamma^{+}, x\right)\right) & =\operatorname{Vol}_{d+1}\left(\tilde{\chi}\left(\Gamma^{+}, x\right)\right) \\
& =\int_{0}^{T}\left(-\partial_{t} u\right) \operatorname{Vol}_{d}\left(I_{u}(x, t)\right) 1_{(x, t) \in \Gamma^{+}} \mathrm{d} t .
\end{aligned}
$$

For any fixed $p \in I(x, t),(x, t) \in \Gamma^{+}$, set

$$
w(y, s)=u(y, s)-p \cdot y .
$$

Then $I_{w}(x, t)=I_{u}(x, t)+p$. Since $w(x, t)-w\left(x \pm e_{i}, t\right) \geq \mp q_{i}$ for any $q \in I_{w}(x, t)$, $i=1, \ldots d$, we have

$$
\operatorname{Vol}_{d}\left(I_{u}(x, t)\right)=\operatorname{Vol}_{d}\left(I_{w}(x, t)\right) \leq \prod_{i=1}^{d}\left[2 u(x, t)-u\left(x+e_{i}, t\right)-u\left(x-e_{i}, t\right)\right]
$$

This inequality, together with (54), yields

$$
\begin{aligned}
& \operatorname{Vol}_{d+1}\left(\chi\left(\Gamma^{+}, x\right)\right) \\
& \leq-C \int_{0}^{T} \partial_{t} u \prod_{i=1}^{d} a_{t}\left(x, x+e_{i}\right)\left[2 u(x, t)-u\left(x+e_{i}, t\right)-u\left(x-e_{i}, t\right)\right] 1_{(x, t) \in \Gamma^{+}} \mathrm{d} t
\end{aligned}
$$




$$
\leq C \int_{0}^{T}\left[-\mathcal{L}_{a} u(x, t)\right]^{d+1} 1_{(x, t) \in \Gamma^{+}} \mathrm{d} t .
$$

Display (53) is proved. Finally, by (52), (53) and $\operatorname{Vol}_{d+1}(\Lambda)=C M^{d+1} / R^{d}$, we conclude that $M^{d+1} / R^{d} \leq C \int_{\Gamma^{+}} f^{d+1}$. The theorem follows.

\section{A.4 Mean value inequality}

Theorem A.4.1 Let $\theta>0$ and $a \in \Omega_{\kappa}$. Recall $\|\cdot\|_{\mathscr{D}, p}$ in (25). For any $\theta_{1} \in(0, \theta)$, $\rho \in(0,1)$ and $p>0$, there exists $C=C\left(\kappa, d, p, \theta, \theta_{1}, \rho\right)$ such that for any function $u$ that solves $\mathcal{L}_{a} u \geq 0$ in $\mathscr{D}=B_{R} \times\left[0, \theta R^{2}\right)$, we have

$$
\sup _{B_{\rho R} \times\left[0, \theta_{1} R^{2}\right)} u \leq C\left\|u^{+}\right\|_{\mathscr{D}, p}
$$

Proof Since $\left\|u^{+}\right\|_{\mathscr{D}, p}$ is increasing in $p>0$, it suffices to consider $p \in(0,1)$. Let $\beta \geq 2$ be a constant to be determined, and set

$$
\eta(x):=\left(1-\frac{|x|^{2}}{R^{2}}\right)^{\beta} 1_{x \in B_{R}}, \quad \zeta(t):=\left(1-\frac{t}{\theta R^{2}}\right)^{\beta} 1_{0 \leq t<\theta R^{2}}
$$

and set $v=\eta u^{+}, \bar{v}=v \zeta$. Define an elliptic operator $L_{a}^{E}$ to be

$$
L_{a}^{E} f(x, t)=\sum_{y: y \sim x} a_{t}(x, y)(f(y, t)-f(x, t))
$$

so that $\mathcal{L}_{a}=L_{a}^{E}+\partial_{t}$. Note that $\left.\bar{v}\right|_{\partial^{\mathrm{P}} \mathscr{D}}=0$ and $\bar{v}(\hat{x})>0$ for $\hat{x} \in \Gamma^{+}(\bar{v}, \mathscr{D})$. (Recall the notation $\Gamma^{+}$above Theorem A.3.1.) By the same argument as in [19, displays (27),(28) and (29)], we have that on $\Gamma^{+}(\bar{v}, \mathscr{D}), u^{+}=u$ and

$$
L_{a}^{E} v \geq \eta L_{a}^{E} u-C_{\beta} \eta^{1-2 / \beta} R^{-2} u^{+} .
$$

Hence, for $X=(x, t) \in \Gamma^{+}(\bar{v}, \mathscr{D})$,

$$
\begin{aligned}
\mathcal{L}_{a} \bar{v} & =\zeta L_{a}^{E} v+\partial_{t}(\zeta \eta u) \\
& \geq \zeta \eta L_{a}^{E} u-C_{\beta} \zeta \eta^{1-2 / \beta} R^{-2} u^{+}+\eta u \partial_{t} \zeta+\zeta \eta \partial_{t} u \\
& =\zeta \eta \mathcal{L}_{a} u-C_{\beta} \zeta \eta^{1-2 / \beta} R^{-2} u^{+}+\eta u \partial_{t} \zeta \\
& \geq-C_{\beta} \zeta \eta^{1-2 / \beta} R^{-2} u^{+}+\eta u \partial_{t} \zeta .
\end{aligned}
$$

Noting that in $\mathscr{D}, \partial_{t} \zeta \geq-C \beta R^{-2} \zeta^{1-1 / \beta} / \theta$ and $\zeta, \eta \in[0,1]$, we have

$$
\mathcal{L}_{a} \bar{v} \geq-C(\eta \zeta)^{1-2 / \beta} R^{-2} u^{+} \quad \text { in } \Gamma^{+}(\bar{v}, \mathscr{D}) .
$$


Applying Theorem A.3.1 to $\bar{v}$ and taking $\beta=2(d+1) / p$,

$$
\begin{aligned}
\sup _{\mathscr{D}} \bar{v} & \leq C\left\|(\eta \zeta)^{1-2 / \beta} u^{+} / \varepsilon\right\|_{\mathscr{D}, d+1} \\
& \leq C\left(\sup _{\mathscr{D}} \bar{v}\right)^{1-p /(d+1)}\left\|\left(u^{+}\right)^{p /(d+1)} / \varepsilon\right\|_{\mathscr{D}, d+1} .
\end{aligned}
$$

Since $\sup _{B_{\rho R} \times\left[0, \theta_{1} R^{2}\right)} u \leq C \sup _{\mathscr{D}} \bar{v}$, the theorem follows.

\section{A.5 Proof of Lemma 25}

Recall $|\mathscr{D}|, \int_{\mathscr{D}},\|\cdot\|_{\mathscr{D}, p}$, and the parabolic cubes in (24), (25) and (26).

Proof First, we claim that there exist constants $\gamma, \delta \in(0,1)$ such that $w(E)>\gamma w(K)$ implies $|E|>\delta|K|$ for all $E \subset K$ where $K \neq \emptyset$ is a subcube of $K^{0}$. Indeed, this is a simple consequence of Hölder's inequality:

$$
\frac{1}{|K|} w(E)=\frac{1}{|K|} \int_{K} w \mathbb{1}_{E} \leq\left(\frac{|E|}{|K|}\right)^{1 / q^{\prime}}\|w\|_{K, q} \stackrel{\left(R H_{q}\right)}{\leq} C\left(\frac{|E|}{|K|}\right)^{1 / q^{\prime}} \frac{w(K)}{|K|},
$$

where $q^{\prime}=q /(q-1)$ denotes the conjugate of $q$.

Assume $K^{0}=K_{r}$. Let $\mathcal{M}_{k}\left(K_{r}\right), k>1$ be the family of nonempty subcubes of $K_{r}$ of the form

$$
\left(\prod_{i=1}^{d}\left[\frac{m_{i}}{2^{k}} r, \frac{1+m_{i}}{2^{k}} r\right) \cap \mathbb{Z}^{d}\right) \times\left[\frac{n_{i}}{4^{k}} r^{2}, \frac{1+n_{i}}{4^{k}} r^{2}\right)
$$

where $m_{i}, n_{i}$ 's are integers. Elements in $M_{k}\left(K_{r}\right)$ are called $k$-level dyadic subcubes of $K_{r}$. Note that every $k$-level cube $K$ is contained in a unique $(k-1)$-level "parent" denoted by $K^{-1}$. Since the class $A_{p}$ is invariant under constant multiplication, we may assume that $w\left(K^{0}\right) /\left|K^{0}\right|=1$.

Let $f:=w^{-1} \mathbb{1}_{K^{0}}$ and define a maximal function

$$
M_{f}(x):=\sup _{K \ni x} \frac{1}{w(K)} \sum_{K}|f| w
$$

where the supremum is taken over all dyadic subcubes $K$ of $K_{0}$. Consider the level sets

$$
E_{k}=\left\{x \in K^{0}: M_{f}(x)>2^{N k}\right\}, \quad k=0,1,2, \ldots
$$

where $N$ is a big constant to be determined. Notice that by assumption, $E_{0}$ is comprised of dyadic subcubes strictly smaller than $K_{0}$. Since $w$ is volume-doubling, there exists a constant $c_{0}>0$ such that for any maximal dyadic subcube $K$ of $E_{k-1}$,

$$
\int_{K} f w \leq \int_{K^{-1}} f w \leq 2^{N(k-1)} w\left(K^{-1}\right) \leq 2^{N(k-1)+c_{0}} w(K) .
$$


Moreover, for the same $K$, we have $2^{N k} w\left(E_{k} \cap K\right) \leq \int_{K} f w$ and so, by the inequality above, $w\left(E_{k} \cap K\right) \leq 2^{c_{0}-N} w(K)$. We now take $N$ to be large enough that $w\left(E_{k} \cap K\right) \leq$ $(1-\gamma) w(K)$ which implies $\left|E_{k} \cap K\right| \leq(1-\delta)|K|$. Summing over all such $K$ 's, we have $\left|E_{k}\right| \leq(1-\delta)\left|E_{k-1}\right|, k \geq 1$. Thus

$$
\left|E_{k}\right| \leq \delta^{k}\left|E_{0}\right| \leq \delta^{k}\left|K^{0}\right|, \quad k=0, \ldots
$$

and so, for $p>1$ chosen so that $p^{\prime}=p /(p-1)$ is sufficiently close to 1 ,

$$
\begin{aligned}
\int_{K^{0}} f^{p^{\prime}-1} & =\int_{K^{0} \cap\left\{x: M_{f} \leq 1\right\}} f^{p^{\prime}-1}+\sum_{k=0}^{\infty} \int_{E_{k} \backslash E_{k+1}} f^{p^{\prime}-1} \\
& \leq\left|K^{0}\right|+\sum_{k=0}^{\infty} 2^{\left(p^{\prime}-1\right) N(k+1)} \delta^{k}\left|K^{0}\right| \\
& \leq C\left|K^{0}\right| .
\end{aligned}
$$

(i) is proved. (ii) then follows from Hölder's inequality

$$
\frac{1}{w(K)} \int_{E} w^{-1} w \leq\left(\frac{1}{w(K)} \int_{K} w^{-p^{\prime}} w\right)^{1 / p^{\prime}}\left(\frac{1}{w(K)} \int_{K} \mathbb{1}_{E} w\right)^{1 / p}
$$

and the $A_{p}$ inequality.

\section{A.6 Proof of Corollary 12(ii)(iii)}

Proof (ii) For any $\hat{x}=(x, t) \in \mathbb{R}^{d} \times[0, \infty)$ and $\omega \in \Omega_{\kappa}$, set

$$
v(\hat{x})=q^{\omega}(\hat{0} ;\lfloor x\rfloor, t) \quad \text { and } \quad a^{\omega}(x):=\int_{0}^{\infty}(v(0, t)-v(x, t)) \mathrm{d} t .
$$

When $d=2$, it suffices to consider $x \in \mathbb{B}_{1} \backslash\{0\}$. We fix a small number $\epsilon \in(0,1)$ and split the integral $a^{\omega}(n x)$ into four parts:

$$
a^{\omega}(n x)=\int_{0}^{n^{\epsilon}}+\int_{n^{\epsilon}}^{n^{2}}+\int_{n^{2}}^{\infty}=: \mathrm{I}+\mathrm{II}+\mathrm{III}
$$

where it is understood that the integrand is $(v(0, t)-v(x, t)) \mathrm{d} t$.

First, we will show that $\mathbb{P}$-almost surely,

$$
\varlimsup_{n \rightarrow \infty}|\mathrm{I}| / \log n \leq \epsilon .
$$


By Theorem 11, for any $t \in\left(0, n^{\epsilon}\right), x \in \mathbb{Z}^{2} \backslash\{0\}$ and all $n$ large enough, $v(n x, t) \leq$ $C e^{-c n|x|} / \rho_{\omega}\left(B_{\sqrt{t}}, 0\right)$. Thus

$$
\int_{0}^{n^{\epsilon}} v(n x, t) \mathrm{d} t \leq \frac{n^{\epsilon}}{\rho_{\omega}(\hat{0})} e^{-c n|x|}
$$

By (i), there exists $t_{0}(\omega)>0$ such that for $n$ big enough with $n^{\epsilon}>t_{0}$,

$$
\int_{0}^{n^{\epsilon}} v(0, t) \mathrm{d} t \leq \frac{C t_{0}}{\rho_{\omega}(\hat{0})}+\int_{t_{0}}^{n^{\epsilon}} \frac{C}{t} \mathrm{~d} t \leq \frac{C t_{0}}{\rho_{\omega}(\hat{0})}+C \epsilon \log n
$$

Display (55) follows immediately.

In the second step, we will show that (note that $2 p_{1}^{\Sigma}(0,0)=1 / \pi \sqrt{\operatorname{det} \Sigma}$ )

$$
\limsup _{n \rightarrow \infty}\left|\mathrm{II}-2 p_{1}^{\Sigma}(0,0) \log n\right| / \log n \leq C \epsilon, \quad \mathbb{P} \text {-a.s. }
$$

Indeed, by Theorem 4, there exists $C(\omega, \epsilon)>0$ such that $\left|t v(0, t)-p_{1}^{\Sigma}(0,0)\right| \leq \epsilon$ whenever $t \geq C(\omega, \epsilon)$. Now, taking $n$ large enough such that $n^{\epsilon}>C(\omega, \epsilon)$,

$$
\begin{aligned}
& \left|\int_{n^{\epsilon}}^{n^{2}} v(0, t) \mathrm{d} t-(2-\epsilon) p_{1}^{\Sigma}(0,0) \log n\right| \\
& \leq \int_{n^{\epsilon}}^{n^{2}}\left|\frac{t v(0, t)-p_{1}^{\Sigma}(0,0)}{t}\right| \mathrm{d} t \\
& \leq \epsilon \int_{n^{\epsilon}}^{n^{2}} \frac{\mathrm{d} t}{t}<2 \epsilon \log n .
\end{aligned}
$$

On the other hand, for $t \geq n^{\epsilon}>t_{0}(\omega)$, by (i), $v(n x, t) \leq \frac{C}{t}\left(e^{-c n|x|}+e^{-c n^{2}|x|^{2} / t}\right)$. Thus

$$
\int_{n^{\epsilon}}^{n^{2}} v(n x, t) \mathrm{d} t \leq \int_{n^{\epsilon}}^{n^{2-\epsilon}} \frac{C}{t} e^{-c n^{\epsilon}|x|^{2}} \mathrm{~d} t+\int_{n^{2-\epsilon}}^{n^{2}} \frac{C}{t} \mathrm{~d} t \leq C \epsilon \log n
$$

Displays (57) and (58) imply (56).

Finally, we will prove that for $\mathbb{P}$-almost every $\omega$,

$$
\limsup _{n \rightarrow \infty}|\mathrm{III}| / \log n=0
$$

Since $|x|<1$, by (44), for any $t \geq n^{2} \geq t_{0}(\omega)$,

$$
|v(0, t)-v(n x, t)| \leq C\left(\frac{n}{\sqrt{t}}\right)^{\gamma} t^{-1} .
$$


Therefore, $\mathbb{P}$-almost surely, when $n^{2}>t_{0}(\omega)$,

$$
\left|\int_{n^{2}}^{\infty} v(0, t)-v(n x, t) \mathrm{d} t\right| \leq C n^{\gamma} \int_{n^{2}}^{\infty} \frac{1}{t^{\gamma / 2+1}} \mathrm{~d} t \leq C
$$

Display (59) follows. Combining (55), (56) and (59), we have for $d=2$,

$$
\varlimsup_{n \rightarrow \infty}\left|\frac{a^{\omega}(n x)}{\log n}-2 p_{1}^{\Sigma}(0,0)\right| \leq C \epsilon
$$

Noting that $\epsilon>0$ is arbitrary, we obtain Corollary 12(ii).

(iii) We fix a small constant $\epsilon \in(0,1)$. Note that

$$
n^{d-2} \int_{0}^{\infty} q^{\omega}(\hat{0} ;\lfloor n x\rfloor, t) \mathrm{d} t=\int_{0}^{\infty} n^{d} v\left(n x, n^{2} s\right) \mathrm{d} s .
$$

For any fixed $x \in \mathbb{R}^{d}$, write

$$
\int_{0}^{\infty} n^{d} v\left(n x, n^{2} s\right) \mathrm{d} s=\int_{0}^{n^{-\epsilon}}+\int_{n^{-\epsilon}}^{\epsilon}+\int_{\epsilon}^{1 / \sqrt{\epsilon}}+\int_{1 / \sqrt{\epsilon}}^{\infty}=: \mathrm{I}+\mathrm{II}+\mathrm{III}+\mathrm{IV} .
$$

First, by Theorem 11, for $s \in\left(0, n^{-\epsilon}\right)$, we have $v\left(n x, n^{2} s\right) \leq C e^{-c n^{\epsilon}|x|^{2}} / \rho_{\omega}(\hat{0})$, hence

$$
\varlimsup_{n \rightarrow \infty} \mathrm{I} \leq C \lim _{n \rightarrow \infty} n^{d-\epsilon} e^{-c n^{\epsilon}|x|^{2}} / \rho_{\omega}(\hat{0})=0 .
$$

Second, by (i), when $n$ is large enough, then for all $t \geq n^{2-\epsilon}$, we have $v(n x, t) \leq$ $C t^{-d / 2} e^{-c n^{2}|x|^{2} / t}$. Hence

$$
\varlimsup_{n \rightarrow \infty} \mathrm{II} \leq \varlimsup_{n \rightarrow \infty} C n^{d} \int_{n^{-\epsilon}}^{\epsilon}\left(n^{2} s\right)^{-d / 2} e^{-c|x|^{2} / s} \mathrm{~d} s \leq C \epsilon .
$$

Moreover, by Theorem 4, there exists $N(\omega, \epsilon)$ such that for $n \geq N(\omega, \epsilon)$, we have $\sup _{|s| \geq \epsilon}\left|v\left(n x, n^{2} s\right)-p_{s}^{\Sigma}(0, x)\right| \leq \epsilon$. Hence

$$
\varlimsup_{n \rightarrow \infty}\left|\mathrm{III}-\int_{\epsilon}^{1 / \sqrt{\epsilon}} p_{s}^{\Sigma}(0, x) \mathrm{d} s\right| \leq \sqrt{\epsilon} .
$$

Further, by (i), for $d \geq 3$,

$$
\varlimsup_{n \rightarrow \infty} \mathrm{IV} \leq C \int_{1 / \sqrt{\epsilon}}^{\infty} \frac{n^{d}}{\left(n^{2} s\right)^{d / 2}} \mathrm{~d} s=C \epsilon^{(d-2) / 4}
$$


Finally, combining (60),(61), (62) and (63), we get

$$
\varlimsup_{n \rightarrow \infty}\left|\int_{0}^{\infty} n^{d} v\left(n x, n^{2} s\right) \mathrm{d} s-\int_{\epsilon}^{1 / \sqrt{\epsilon}} p_{s}^{\Sigma}(0, x) \mathrm{d} s\right| \leq C \epsilon^{1 / 4}
$$

Letting $\epsilon \rightarrow 0$, (iii) is proved.

\section{References}

1. Andres, S.: Invariance principle for the random conductance model with dynamic bounded conductances. Ann. Inst. Henri Poincaré Probab. Stat. 50(2), 352-374 (2014)

2. Andres, S., Chiarini, A., Deuschel, J.-D., Slowik, M.: Quenched invariance principle for random walks with time-dependent ergodic degenerate weights. Ann. Probab. 46(1), 302-336 (2018)

3. Andres, S., Chiarini, A., Slowik, M.: Quenched local limit theorem for random walks among timedependent ergodic degenerate weights. Probab. Theory Related Fields 179, 1145-1181 (2021)

4. Andres, S., Deuschel, J.-D., Slowik, M.: Harnack inequalities on weighted graphs and some applications to the random conductance model. Probab. Theory Related Fields 164(3-4), 931-977 (2016)

5. Andres, S., Deuschel, J.-D., Slowik, M.: Green kernel asymptotics for two-dimensional random walks under random conductances. Electron. Commun. Probab. 25(58), 1-14 (2020)

6. Bauman, P.: Positive solutions of elliptic equations in nondivergence form and their adjoints. Ark. Mat. 22(2), 153-173 (1984)

7. Barlow, M., Hambly, B.: Parabolic Harnack inequality and local limit theorem for percolation clusters. Electron. J. Probab. 14(1), 1-27 (2009)

8. Berger, N., Criens, D.: A parabolic Harnack inequality for balanced random environments. Work in progress

9. Berger, N., Deuschel, J.-D.: A quenched invariance principle for non-elliptic random walk in i.i.d. balanced random environment. Probab. Theory Related Fields 158(1-2), 91-126 (2014)

10. Coifman, R., Fefferman, C.: Weighted norm inequalities for maximal functions and singular integrals. Studia Math. 51, 241-250 (1974)

11. Criens, D.: Essays on Stochastic Processes and their Applications. PhD Thesis, (2020)

12. Delmotte, T.: Parabolic Harnack inequality and estimates of Markov chains on graphs. Rev. Mat. Iberoam. 15, 181-232 (1999)

13. Delmotte, T., Deuschel, J.-D.: On estimating the derivatives of symmetric diffusions in stationary random environment, with applications to $\nabla \varphi$ interface model. Probab. Theory Relat. Fields 133(3), 358-390 (2005)

14. Deuschel, J.-D., Guo, X., Ramirez, A.: Quenched invariance principle for random walk in timedependent balanced random environment. Ann. Inst. Henri Poincaré Probab. Stat. 54(1), 363-384 (2018)

15. Escauriaza, L.: Bounds for the fundamental solutions of elliptic and parabolic equations: In memory of Eugene Fabes. Commun. Part. Differ. Equ. 25(5-6), 821-845 (2000)

16. Fabes, E., Safonov, M., Yuan, Y.: Behavior near the boundary of positive solutions of second order parabolic equations. II. Trans. Am. Math. Soc. 351(12), 4947-4961 (1999)

17. Fabes, E., Stroock, D.: The $L^{p}$-integrability of Green's functions and fundamental solutions for elliptic and parabolic equations. Duke Math. J. 51(4), 997-1016 (1984)

18. Garofalo, N.: Second order parabolic equations in nonvariational form: boundary Harnack principle and comparison theorems for nonnegative solutions. Annali di matematica pura ed applicata 138(1), 267-296 (1984)

19. Guo, X., Zeitouni, O.: Quenched invariance principle for random walks in balanced random environment. Probab. Theory Relat. Fields 152, 207-230 (2012)

20. Huang, R., Kumagai, T.: Stability and instability of Gaussian heat kernel estimates for random walks among time-dependent conductances, Electron. Commun. Probab. 21, paper no. 5 (2016)

21. Krengel, U., Pyke, R.: Uniform pointwise ergodic theorems for classes of averaging sets and multiparameter subadditive processes. Stochastic Process. Appl. 26(2), 289-296 (1987) 
22. Krylov, N., Safonov, M.: A property of the solutions of parabolic equations with measurable coefficients. Izv. Akad. Nauk SSSR Ser. Mat. 44(1), 161-239 (1980)

23. Kuo, H.-J., Trudinger, N.: Evolving monotone difference operators on general space-time meshes. Duke Math. J. 91(3), 587-607 (1998)

24. Lawler, G.: Weak convergence of a random walk in a random environment. Comm. Math. Phys. 87(1), 81-87 (1982/83)

25. Lawler, G.: Estimates for differences and Harnack inequality for difference operators coming from random walks with symmetric, spatially inhomogeneous increments. Proc. Lond. Math. Soc. 63(3), 552-568 (1991)

26. Mustapha, S.: Gaussian estimates for spatially inhomogeneous random walks on $\mathbb{Z}^{d}$. Ann. Probab. 34(1), 264-283 (2006)

27. Papanicolaou, G., Varadhan, S.R.S.: Diffusions with random coefficients. Statistics and probability: essays in honor of C. R. Rao, pp. 547-552, North-Holland, Amsterdam, (1982)

28. Safonov, M., Yuan, Y.: Doubling properties of second order parabolic equations. Ann. Math. Second Ser. 150(1), 313-328 (1999)

29. Stein, E.: Harmonic analysis: real-variable methods, orthogonality, and oscillatory integrals. Princeton Mathematical Series, 43. Monographs in Harmonic Analysis, III. Princeton University Press, Princeton, NJ (1993)

Publisher's Note Springer Nature remains neutral with regard to jurisdictional claims in published maps and institutional affiliations. 